\title{
Penning-Trap Searches for Lorentz and CPT Violation
}

\author{
Yunhua Ding ${ }^{1,2, *}$, Teague D. Olewiler ${ }^{2}$ and Mohammad Farhan Rawnak ${ }^{2}$ \\ 1 W.M. Keck Science Department, Claremont McKenna, Pitzer, and Scripps Colleges, \\ Claremont, CA 91711, USA \\ 2 Department of Physics, Gettysburg College, Gettysburg, PA 17325, USA; olewte01@gettysburg.edu (T.D.O.); \\ rawnmo01@gettysburg.edu (M.F.R.) \\ * Correspondence: yding@kecksci.claremont.edu
}

\section{check for}

updates

Citation: Ding, Y.; Olewiler, T.D.; Rawnak, M.F. Penning-Trap Searches for Lorentz and CPT Violation. Symmetry 2021, 13, 1703. https:// doi.org/10.3390/sym13091703

Academic Editor: Matthew Mewes

Received: 27 August 2021

Accepted: 14 September 2021

Published: 15 September 2021

Publisher's Note: MDPI stays neutral with regard to jurisdictional claims in published maps and institutional affiliations.

Copyright: (c) 2021 by the authors. Licensee MDPI, Basel, Switzerland. This article is an open access article distributed under the terms and conditions of the Creative Commons Attribution (CC BY) license (https:// creativecommons.org/licenses/by/ $4.0 /)$.

\begin{abstract}
An overview of recent progress on testing Lorentz and CPT symmetry using Penning traps is presented. The theory of quantum electrodynamics with Lorentz-violating operators of mass dimensions up to six is summarized. Dominant shifts in the cyclotron and anomaly frequencies of the confined particles and antiparticles due to Lorentz and CPT violation are derived. Existing results of the comparisons of charge-to-mass ratios and magnetic moments involving protons, antiprotons, electrons, and positrons are used to constrain various coefficients for Lorentz violation.
\end{abstract}

Keywords: Lorentz and CPT violation; Penning trap; standard-model extension

\section{Introduction}

Precision experiments involving Penning traps have in recent years achieved impressive sensitivities to properties of fundamental particles. For example, the magnetic moment of electrons has been measured to a record precision of $0.28 \mathrm{ppt}$ [1]. The high precision offered by Penning-trap experiments provides excellent opportunities to test fundamental symmetries. This includes the Lorentz symmetry, one of the foundations of both general relativity and the Standard Model of particle physics. It has been recently shown that tiny violations of Lorentz symmetry could naturally arise in a fundamental theory that unifies gravity with quantum physics at the Plank scale $M_{P} \sim 10^{19} \mathrm{GeV}$, such as string theory [2,3]. As in any effective field theory, violations of CPT symmetry also break Lorentz symmetry [4-6], testing Lorentz symmetry also includes CPT tests. In recent years, searches for Lorentz and CPT violation in precision experiments have been performed across many subfields of physics [7], including Penning-trap experiments. Here, we provide an overview of the recent progress on testing Lorentz and CPT violation in Penning-trap experiments measuring charge-to-mass ratios and magnetic moments of protons, antiprotons, electrons, and positrons.

In the context of effective field theory, the comprehensive framework that describes all possible Lorentz violation is the Standard Model Extension (SME) [4,5,8]. The Lagrange density of the SME is constructed from general relativity and the Standard Model by adding all possible Lorentz-violating terms. Each of such terms is formed from a coordinate-independent contraction of a general Lorentz-violating operator with a corresponding coefficient. The subset of the SME with operators of mass dimensions $d \leq 4$ is called the minimal SME, which is power-counting renormalizable. For the non-minimal $\mathrm{SME}$, it restricts attention to operators of mass dimensions $d>4$, which is viewed to produce higher-order effects. Study of the non-minimal SME serves as a basis for further investigations of many aspects of Lorentz and CPT violation, such the causality and stability [9,10], Lorentz-violating models in supersymmetry [11], non-commutative Lorentzviolating quantum electrodynamics [12-14], and the underlying pseudo-Riemann-Finsler geometry [15-18].

For Penning-trap experiments measuring charge-to-mass ratios and magnetic moments of confined particles or antiparticles, both the minimal and nonminimal SME can 
produce various measurable Lorentz- and CPT-violating effects via shifts in the cyclotron and anomaly frequencies. These effects in general can depend on sidereal time and differ between particles and antiparticles. In the minimal SME, the authors of $[19,20]$ present the first theoretical analysis to study Lorentz and CPT violation in Penning traps. An extension to the nonminimal SME by including Lorentz-violating operators of mass dimensions up to six was recently made in [21], in which analysis of the magnetic moment comparisons between particles and antiparticles using Penning traps was also performed. A similar application to charge-to-ratio comparisons is presented in [22]. For the effects arising from sidereal variations due to the Earth's rotation, the related discussions are given in [23-25].

In this work, we provide an overview of recent progress on searching for Lorentz- and CPT-violating signals using Penning traps and provide the most updated constraints on the coefficients for Lorentz violation that are relevant to these experiments. The results provided in this work are complementary to these from the studies of Lorentz and CPT violation in experiments involving measurements of the muon's anomalous magnetic moment [26,27], the spectroscopic analysis of hydrogen, antihydrogen, and other related systems [28], and clock comparisons [29].

This work is organized as follows. We start in Section 2 with the related theory, where we present in Section 2.1 the theory of Lorentz-violating quantum electrodynamics with operators of mass dimensions up to six. The perturbative energy shifts to the confined particles or antiparticles are obtained in Section 2.2 using perturbation theory. Section 2.3 gives the corresponding shifts in the cyclotron and anomaly frequencies due to Lorentz and CPT violation. The discussion of sidereal variations and rotation matrices are given in Section 2.4. Then, we turn in Section 3 to experimental applications to various Penningtrap experiments and present the constraints on the coefficients for Lorentz violation. The applications to charge-to-mass ratio comparisons are treated in Section 3.1, with Section 3.1.1 focusing on the proton sector, and Section 3.1.2 discussing the electron sector, respectively. The resulting constraints on the coefficients for Lorentz violation from the reported experimental results are summarized in Tables 1-3. Section 3.2 discusses the applications to magnetic moment comparisons, with Section 3.2.1 for the proton sector, and Section 3.2.2 for the electron sector, respectively. The corresponding limits on the coefficients for Lorentz violation are listed in Tables 4 and 5. The summary of this work is given in Section 4. Finally, Appendix A lists the explicit expressions of the transformations of the relevant coefficients into different frames. Throughout this work, we follow the same notation used in $[21,22]$ and adopt natural units with $\hbar=c=1$.

\section{Theory}

In this section, we summarize the theory developed in [21] of Lorentz-violating quantum electrodynamics with operators of mass dimension $d \leq 6$ and derive the energy shifts for particles and antiparticles confined in Penning traps due to Lorentz and CPT violation.

\subsection{Lagrange Density}

For a single Dirac fermion field $\psi$ of charge $q$ and mass $m_{\psi}$, the general Lorentzviolating Lagrange density $\mathcal{L}_{\psi}$ can be constructed by adding a general Lorentz-violating operator $\widehat{\mathcal{Q}}$ to the conventional Lagrange density,

$$
\mathcal{L}_{\psi}=\frac{1}{2} \bar{\psi}\left(\gamma^{\mu} i D_{\mu}-m_{\psi}+\widehat{\mathcal{Q}}\right) \psi+\text { H.c., }
$$

where $D_{\mu}=\left(\partial_{\mu}+i q A_{\mu}\right)$ is the covariant derivative given by the minimal coupling with $A_{\mu}$ being the electromagnetic four-potential. H.c. means Hermitian conjugate. The general Lorentz-violating operator $\widehat{\mathcal{Q}}$ in the Lagrange density (1) is a $4 \times 4$ spinor matrix that contains terms formed by the contraction of a generic coefficient for Lorentz violation, the covariant derivative $i D_{\mu}$, the antisymmetric electromagnetic field tensor $F_{\alpha \beta} \equiv \partial_{\alpha} A_{\beta}-\partial_{\beta} A_{\alpha}$, and one of the 16 Dirac matrix bases. For example, one of the dimension-five operators involving the $F$-type coefficients for Lorentz violation takes the form $b_{F}^{(5) \mu \alpha \beta} F_{\alpha \beta} \gamma_{5} \gamma_{\mu}$. For 
mass dimension $d \leq 6$, a full list of the relevant coefficients for Lorentz violation and their properties are given in Table I in [21]. Note that the hermiticity of the Lagrange density (1) indicates that the operator $\widehat{\mathcal{Q}}$ satisfies the condition $\widehat{\mathcal{Q}}=\gamma_{0} \widehat{\mathcal{Q}}^{\dagger} \gamma_{0}$. In the free-fermion limit where $A_{\alpha}=0$, the explicit expression of the Lagrange density (1) at arbitrary mass dimension has been studied in [30]. For the interaction case where $A_{\alpha} \neq 0$, [21] developed a theory for operators of mass dimensions up to six. An extension of the theory to include operators of arbitrary mass dimension was recently given in [31]. Similar analysis has also been performed for other SME sectors, including these for photon [32], neutrino [33], and gravity [34].

Due to the existence of the general operator $\widehat{\mathcal{Q}}$ in the Lagrange density (1), the conventional Dirac equation for a fermion in electromagnetic fields is modified to

$$
\left(p \cdot \gamma-m_{\psi}+\widehat{\mathcal{Q}}\right) \psi=0
$$

where we have chosen the momentum space for convenience, with the identification $p_{\alpha} \leftrightarrow i D_{\alpha}$. Given the fact that no Lorentz-violating signals have been observed so far, any such signal must be tiny compared to the energy scale of the system of interest. Therefore, we can treat the corrections due to Lorentz and CPT violation to the conventional Hamiltonian as perturbative and apply perturbation theory to obtain the dominant shifts to the energy levels of the confined particles and antiparticles. From Equation (2), the exact Hamiltonian $\mathcal{H}$ can be defined as

$$
\mathcal{H} \psi \equiv p^{0} \psi=\gamma_{0}\left(\boldsymbol{p} \cdot \gamma+m_{\psi}-\widehat{\mathcal{Q}}\right) \psi=\left(\mathcal{H}_{0}+\delta \mathcal{H}\right) \psi
$$

where $p^{0}$ is the exact energy of the system of interest, including all contributions from Lorentz and CPT violation, $\mathcal{H}_{0}$ is the conventional Hamiltonian for a fermion in an electromagnetic field, and $\delta \mathcal{H}=-\gamma_{0} \widehat{\mathcal{Q}}$ is the exact perturbative Hamiltonian.

To construct $\delta \mathcal{H}$, we note that operator $\widehat{\mathcal{Q}}$ in general contains terms that are of powers of $p^{0}$, which corresponds to the exact Hamiltonian $\mathcal{H}$ itself. In certain simple cases, one can apply a field redefinition to remove the additional time derivatives and adopt the standard procedure involving time translation on wave functions to obtain the exact perturbative Hamiltonian $\delta \mathcal{H}$ [20]. In more general cases, it is challenging to directly construct $\delta \mathcal{H}$ due to the existence of powers of time derivatives. However, we notice that any contributions to $\delta \mathcal{H}$ due to the exact Hamiltonian $\mathcal{H}$ are at second or higher orders in the coefficients for Lorentz violation. Therefore, to obtain the leading-order results, one can apply the following substitution $[21,33]$ :

$$
\delta \mathcal{H} \approx-\left.\gamma_{0} \widehat{\mathcal{Q}}\right|_{p^{0} \rightarrow E_{0}}
$$

where $E_{0}$ is the unperturbative eigenvalue, which can be obtained by solving the conventional Dirac equation for a fermion in an electromagnetic field.

\subsection{Perturbative Energy Shifts}

With the perturbative Hamiltonian $\delta \mathcal{H}$ determined by Equation (4), the shifts in the energy levels of a confined particle can be obtained by applying perturbation theory,

$$
\delta E_{n, \pm}=\left\langle\chi_{n, \pm}|\delta \mathcal{H}| \chi_{n, \pm}\right\rangle
$$

where $\chi_{n, \pm}$ are the unperturbative stationary eigenstates with $n$ being the level number and \pm denoting the spin for a positive-energy fermion. $\delta E_{n, \pm}$ are the perturbative corrections to the energy levels due to Lorentz and CPT violation.

To derive the expressions for $\delta E_{n, \pm}$, we can take an idealized Penning trap where a constant uniform magnetic field is applied to confine the particle's radial motion and a quadrupole electric field provides the axial confinement. The dominant effects in the unperturbative energy levels are due to the interactions between the confined particle and the magnetic field. The quadruple electric field generates effects suppressed by a 
factor of $E / B \approx 10^{-5}$ in natural units for a typical Penning trap field configuration with $E \approx 20 \mathrm{kV} / \mathrm{m}$ and $B \approx 5 \mathrm{~T}$. Therefore, the leading-order results in $\delta E_{n, \pm}$ can be obtained by further idealizing the trap as a pure uniform magnetic field in which a quantum fermion moves. Following the above discussion, for a spin- $1 / 2$ fermion, the leading-order perturbative energy shifts due to Lorentz and CPT violation are found to be [22]

$$
\begin{aligned}
\delta E_{n, \pm 1}^{w}= & \widetilde{a}_{w}^{0} \mp \sigma \widetilde{b}_{w}^{3}-\widetilde{m}_{F, w}^{3} B \pm \sigma \widetilde{b}_{F, w}^{33} B+\left( \pm \sigma \widetilde{b}_{w}^{3}-m_{w}\left[\widetilde{c}_{w}^{00}+\left(\widetilde{c}_{w}^{11}+\widetilde{c}_{w}^{22}\right)_{s}\right]\right) \frac{(2 n+1 \mp \sigma)|q B|}{2 m_{w}^{2}} \\
& +\left(\mp \sigma\left(\widetilde{b}_{w}^{311}+\widetilde{b}_{w}^{322}\right)-\frac{1}{m_{w}}\left(\widetilde{c}_{w}^{11}+\widetilde{c}_{w}^{22}\right)_{\overparen{s}}\right) \frac{(2 n+1)|q B|}{2},
\end{aligned}
$$

where $\sigma$ is the charge sign of the fermion, given by $q \equiv \sigma|q|$. The subscript $w$ indicates the fermion species. For example, for electrons and protons, $w=e$ and $w=p$, respectively. The tilde coefficients are defined by

$$
\begin{aligned}
\widetilde{a}_{w}^{0}= & a_{w}^{0}-m_{w} c_{w}^{00}-m_{w} e_{w}^{0}+m_{w}^{2} m_{w}^{(5) 00}+m_{w}^{2} a_{w}^{(5) 000}-m_{w}^{3} c_{w}^{(6) 0000}-m_{w}^{3} e_{w}^{(6) 000}, \\
\widetilde{b}_{w}^{3}= & b_{w}^{3}+H_{w}^{12}-m_{w} d_{w}^{30}-m_{w} g_{w}^{120}+m_{w}^{2} b_{w}^{(5) 300}+m_{w}^{2} H_{w}^{(5) 1200}-m_{w}^{3} d_{w}^{(6) 3000}-m_{w}^{3} g_{w}^{(6) 12000}, \\
\widetilde{m}_{F, w}^{3}= & m_{F, w}^{(5) 12}+a_{F, w}^{(5) 012}-m_{w} c_{F, w}^{(6) 0012}-m_{w} e_{F, w}^{(6) 012}, \\
\widetilde{b}_{F, w}^{33}= & b_{F, w}^{(5) 312}+H_{F, w}^{(5) 1212}-m_{w} d_{F, w}^{(6) 3012}-m_{w} g_{F, w}^{(6) 12012}, \\
\widetilde{b}_{w}^{\prime 3}= & b_{w}^{3}+m_{w}\left(g_{w}^{120}-g_{w}^{012}+g_{w}^{021}\right)-m_{w}^{2} b_{w}^{(5) 300}-2 m_{w}^{2}\left(H_{w}^{(5) 1200}-H_{w}^{(5) 0102}+H_{w}^{(5) 0201}\right) \\
& +2 m_{w}^{3} d_{w}^{(6) 3000}+3 m_{w}^{3}\left(g_{w}^{(6) 12000}-g_{w}^{(6) 01002}+g_{w}^{(6) 02001}\right), \\
\widetilde{c}_{w}^{00}= & c_{w}^{00}-m_{w} m_{w}^{(5) 00}-2 m_{w} a_{w}^{(5) 000}+3 m_{w}^{2} c_{w}^{(6) 0000}+2 m_{w}^{2} e_{w}^{(6) 000},
\end{aligned}
$$

and the " $11+22$ " types of tilde coefficients are defined by

$$
\begin{aligned}
\left(\widetilde{c}_{w}^{j j}\right)_{s} & =c_{w}^{j j}-2 m_{w} a_{w}^{(5) j 0 j}+3 m_{w}^{2} c_{w}^{(6) j 00 j}, \\
\left(\widetilde{c}_{w}^{j j}\right)_{\widehat{s}} & =-m_{w} a_{w}^{(5) 0 j j}-m_{w} m_{w}^{(5) j j}+3 m_{w}^{2} c_{w}^{(6) 00 j j}+3 m_{w}^{2} e_{w}^{(6) 0 j j}, \\
\widetilde{b}_{w}^{3 j j} & =b_{w}^{(5) 3 j j}+H_{w}^{(5) 12 j j}-3 m_{w} d_{w}^{(6) 30 j j}-3 m_{w} g_{w}^{(6) 120 j j},
\end{aligned}
$$

with $j=1$ or 2 only. The subscripts $s$ and $\bar{s}$ in the $\widetilde{c}_{w}^{j j}$ tilde coefficients in the energy shifts (6) show that $\left(\widetilde{c}_{w}^{j j}\right)_{s}$ give both spin-dependent and spin-independent energy shifts, while $\left(\widetilde{c}_{w}^{j j}\right)_{\bar{s}}$ produce only spin-independent ones, as evident from the corresponding proportional factors $2 n+1 \mp \sigma$ and $2 n+1$. We note that the tilde coefficients $\widetilde{a}_{w}^{0}, \widetilde{b}_{w}^{3}, \widetilde{m}_{F, w}^{3}$, and $\widetilde{b}_{F, w}^{33}$ in the energy shifts (6) produce effects that are independent of the level number $n$.

Following a similar analysis, the corresponding leading-order shifts to the energy levels of antifermions can be determined by using

$$
\delta E_{n, \pm}^{c}=\left\langle\chi_{n, \pm}^{c}\left|\delta \mathcal{H}^{c}\right| \chi_{n, \pm}^{c}\right\rangle,
$$

where $\chi_{n, \pm}^{c}$ are the positive-energy antifermion eigenstates, obtained by applying charge conjugation on the negative-energy fermion solutions $\chi_{n, \pm} . \delta \mathcal{H}^{c}$ is the antifermion perturbative Hamiltonian, which can be obtained from $\delta \mathcal{H}$ by reversing the charge sign $\sigma$ and changing the signs for all CPT-odd coefficients in operator $\widehat{\mathcal{Q}}$. Using Equation (9), the antifermion results are given by [22]

$$
\begin{aligned}
\delta E_{n, \pm 1}^{\overline{\bar{w}}}= & -\widetilde{a}_{w}^{* 0} \pm \sigma \widetilde{b}_{w}^{* 3}-\widetilde{m}_{F, w}^{* 3} B \mp \sigma \widetilde{b}_{F, w}^{* 33} B+\left(\mp \sigma \widetilde{b}_{w}^{* 3}-m_{w}\left[\widetilde{c}_{w}^{00}+\left(\widetilde{c}_{w}^{11}+\widetilde{c}_{w}^{22}\right)_{s}\right]\right) \frac{(2 n+1 \mp \sigma)|q B|}{2 m_{w}^{2}} \\
& +\left( \pm \sigma\left(\widetilde{b}_{w}^{* 311}+\widetilde{b}_{w}^{* 322}\right)-\frac{1}{m_{w}}\left(\widetilde{c}_{w}^{11}+\widetilde{c}_{w}^{22}\right)_{\bar{s}}\right) \frac{(2 n+1)|q B|}{2}
\end{aligned}
$$


where the tilde quantities with a star subscript are given by

$$
\begin{aligned}
\widetilde{a}_{w}^{* 0}= & a_{w}^{0}+m_{w} c_{w}^{00}-m_{w} e_{w}^{0}-m_{w}^{2} m_{w}^{(5) 00}+m_{w}^{2} a_{w}^{(5) 000}+m_{w}^{3} c_{w}^{(6) 0000}-m_{w}^{3} e_{w}^{(6) 000}, \\
\widetilde{b}_{w}^{* 3}= & b_{w}^{3}-H_{w}^{12}+m_{w} d_{w}^{30}-m_{w} g_{w}^{120}+m_{w}^{2} b_{w}^{(5) 300}-m_{w}^{2} H_{w}^{(5) 1200}+m_{w}^{3} d_{w}^{(6) 3000}-m_{w}^{3} g_{w}^{(6) 12000}, \\
\widetilde{m}_{F, w}^{* 3}= & m_{F, w}^{(5) 12}-a_{F, w}^{(5) 012}-m_{w} c_{F, w}^{(6) 0012}+m_{w} e_{F, w}^{(6) 012}, \\
\widetilde{b}_{F, w}^{* 33}= & b_{F, w}^{(5) 312}-H_{F, w}^{(5) 1212}+m_{w} d_{F, w}^{(6) 3012}-m_{w} g_{F, w}^{(6) 12012}, \\
\widetilde{b}_{w}^{\prime * 3}= & b_{w}^{3}+m_{w}\left(g_{w}^{120}-g_{w}^{012}+g_{w}^{021}\right)-m_{w}^{2} b_{w}^{(5) 300}+2 m_{w}^{2}\left(H_{w}^{(5) 1200}-H_{w}^{(5) 0102}+H_{w}^{(5) 0201}\right) \\
& -2 m_{w}^{3} d_{w}^{(6) 3000}+3 m_{w}^{3}\left(g_{w}^{(6) 12000}-g_{w}^{(6) 01002}+g_{w}^{(6) 02001}\right), \\
\widetilde{c}_{w}^{* 00}= & c_{w}^{00}-m_{w} m_{w}^{(5) 00}+2 m_{w} a_{w}^{(5) 000}+3 m_{w}^{2} c_{w}^{(6) 0000}-2 m_{w}^{2} e_{w}^{(6) 000},
\end{aligned}
$$

and the corresponding " $11+22$ " starred tilde quantities are defined by

$$
\begin{aligned}
\left(\widetilde{c}_{w}^{* j j}\right)_{s} & =c_{w}^{j j}+2 m_{w} a_{w}^{(5) j 0 j}+3 m_{w}^{2} c_{w}^{(6) j 00 j}, \\
\left(\widetilde{c}_{w}^{* j j}\right)_{\bar{s}} & =m_{w} a_{w}^{(5) 0 j j}-m_{w} m_{w}^{(5) j j}+3 m_{w}^{2} c_{w}^{(6) 00 j j}-3 m_{w}^{2} e_{w}^{(6) 0 j j}, \\
\widetilde{b}_{w}^{* 3 j j} & =b_{w}^{(5) 3 j j}-H_{w}^{(5) 12 j j}+3 m_{w} d_{w}^{(6) 30 j j}-3 m_{w} g_{w}^{(6) 120 j j} .
\end{aligned}
$$

In the result (10), the charge sign $\sigma$ is understood to be reversed for the antifermion. Comparing the fermion and antifermion energy shifts results (6) and (10), $\delta E_{n, \pm 1}^{\bar{w}}$ can also been obtained from $\delta E_{n, \pm 1}^{w}$ by reversing the charge sign $\sigma$, the spin orientation, and the signs of all CPT-odd coefficients for Lorentz violation, as expected.

We remark in passing that the rotation properties of the coefficients for Lorentz violation appearing in results (6) and (10) are represented by their indices. For example, the index pair " 12 " on the right sides of the definitions (7) and (11) is antisymmetric. This implies that these coefficients for Lorentz violation transform like a single " 3 " index under rotations, while coefficients with an index " 0 " or an index pair " 00 " are invariant under rotations. The cylindrical symmetry of the Penning trap is correctly reflected by the fact that the results (6) and (10) only depend on index " 0 ", " 3 ", and " $11+22$ ".

\subsection{Cyclotron and Anomaly Frequencies}

The primary observables of interest in a Penning-trap experiment are frequencies. Two key frequencies are the cyclotron frequency $v_{c} \equiv \omega_{c} / 2 \pi$ and the Larmor spin-precession frequency $v_{L} \equiv \omega_{L} / 2 \pi$. The difference of the two frequencies gives the anomaly frequency $v_{L}-v_{c}=v_{a} \equiv \omega_{a} / 2 \pi$ [35]. For a confined fermion of flavor $w$ in a Penning trap, the cyclotron and anomaly frequencies are defined as the energy difference between the following energy levels [20,21]:

$$
\omega_{c}^{w}=E_{1, \sigma}^{w}-E_{0, \sigma^{\prime}}^{w}, \quad \omega_{a}^{w}=E_{0,-\sigma}^{w}-E_{1, \sigma}^{w} .
$$

For an antifermion of flavor $\bar{w}$, the corresponding definitions for the cyclotron and anomaly frequencies are given by $[20,21]$

$$
\omega_{c}^{\bar{w}}=E_{1, \sigma}^{\bar{w}}-E_{0, \sigma}^{\bar{w}}, \quad \omega_{a}^{\bar{w}}=E_{0,-\sigma}^{\bar{w}}-E_{1, \sigma^{\prime}}^{\bar{w}}
$$

with the understanding that the charge signs $\sigma$ in definitions (14) are reversed compared to these in definitions (13).

In a Lorentz-invariant scenario, the charge-to-mass ratio and the $g$ factor of a particle or an antiparticle confined in a Penning trap with a magnetic field strength $B$ are related to the above cyclotron and anomaly frequencies by

$$
\frac{|q|}{m}=\frac{\omega_{c}}{B},
$$


and

$$
\frac{g}{2}=\frac{\omega_{L}}{\omega_{c}}=1+\frac{\omega_{a}}{\omega_{c}}
$$

respectively. For Penning-trap experiments comparing the charge-to-mass ratios or the $g$ factors between a particle of species $w$ and its corresponding antiparticle $\bar{w}$, the CPT theorem guarantees that the following differences must be zero,

$$
\frac{(|q| / m)_{\bar{w}}}{(|q| / m)_{w}}-1=\frac{\omega_{c}^{\bar{w}}}{\omega_{c}^{w}}-1=0
$$

and

$$
\frac{1}{2}\left(g_{w}-g_{\bar{w}}\right)=\frac{\omega_{a}^{w}}{\omega_{c}^{w}}-\frac{\omega_{a}^{\bar{w}}}{\omega_{c}^{\bar{w}}}=0,
$$

where in Equation (17), for simplicity, we have assumed the same magnetic field is used in the comparison. When different magnetic fields are used, the expression can be easily obtained by substituting $\omega_{c}^{\bar{w}} / \omega_{c}^{w}$ in the middle term of Equation (17) by $\left(\omega_{c}^{\bar{w}} / B_{\bar{w}}\right)\left(\omega_{c}^{w} / B_{w}\right)$, where $B_{w}$ and $B_{\bar{w}}$ are the strengths of the magnetic fields used to confine the particle and antiparticle, respectively. However, this assumption is not required to derive Equation (18) as the ratios $\omega_{a}^{w} / \omega_{c}^{w}$ and $\omega_{a}^{\bar{w}} / \omega_{c}^{\bar{w}}$ do not depend on the magnetic field used in the trap.

However, in the presence of Lorentz and CPT violation, the picture changes dramatically as both the cyclotron and anomaly frequencies for fermions and antifermions can be shifted due to the Lorentz- and CPT-violating corrections to the energy levels,

$$
\begin{array}{cc}
\delta \omega_{c}^{w}=\delta E_{1, \sigma}^{w}-\delta E_{0, \sigma}^{w}, & \delta \omega_{a}^{w}=\delta E_{0,-\sigma}^{w}-\delta E_{1, \sigma}^{w} \\
\delta \omega_{c}^{\bar{w}}=\delta E_{1, \sigma}^{\bar{w}}-\delta E_{0, \sigma}^{\bar{w}} & \delta \omega_{a}^{\bar{w}}=\delta E_{0,-\sigma}^{\bar{w}}-\delta E_{1, \sigma}^{\bar{w}} .
\end{array}
$$

Applying energy shift results (6) and (10), the corrections to the cyclotron frequencies for a fermion and antifermion are found to be [22]

$$
\begin{aligned}
& \delta \omega_{c}^{w}=\left(\frac{1}{m_{w}^{2}} \widetilde{b}_{w}^{\prime 3}-\frac{1}{m_{w}}\left(\widetilde{c}_{w}^{00}+\widetilde{c}_{w}^{11}+\widetilde{c}_{w}^{22}\right)-\left(\widetilde{b}_{w}^{311}+\widetilde{b}_{w}^{322}\right)\right) e B, \\
& \delta \omega_{c}^{\bar{w}}=\left(-\frac{1}{m_{w}^{2}} \widetilde{b}_{w}^{\prime * 3}-\frac{1}{m_{w}}\left(\widetilde{c}_{w}^{* 00}+\widetilde{c}_{w}^{* 11}+\widetilde{c}_{w}^{* 22}\right)+\left(\widetilde{b}_{w}^{* 311}+\widetilde{b}_{w}^{* 322}\right)\right) e B,
\end{aligned}
$$

where the tilde and starred tilde coefficients are given by definitions (7) and (11). The $\widetilde{c}_{w}^{j j}$ and $\widetilde{c}_{w}^{* j j}$ tilde coefficients with $j$ taking values of 1 or 2 are the sum of the two quantities given in definitions (8) and (12),

$$
\begin{aligned}
\widetilde{c}_{w}^{j j} & =\left(\vec{c}_{w}^{j j}\right)_{s}+\left(\widetilde{c}_{w}^{j j}\right)_{\widehat{s}} \\
& =c_{w}^{j j}-2 m_{w} a_{w}^{(5) j 0 j}+3 m_{w}^{2} c_{w}^{(6) j 00 j}-m_{w} a_{w}^{(5) 0 j j}-m_{w} m_{w}^{(5) j j}+3 m_{w}^{2} c_{w}^{(6) 00 j j}+3 m_{w}^{2} e_{w}^{(6) 0 j j}, \\
\widetilde{c}_{w}^{* j j} & =\left(\widetilde{c}_{w}^{* j j}\right)_{s}+\left(\widetilde{c}_{w}^{* j j}\right)_{\bar{s}} \\
& =c_{w}^{j j}+2 m_{w} a_{w}^{(5) j 0 j}+3 m_{w}^{2} c_{w}^{(6) j 00 j}+m_{w} a_{w}^{(5) 0 j j}-m_{w} m_{w}^{(5) j j}+3 m_{w}^{2} c_{w}^{(6) 00 j j}-3 m_{w}^{2} e_{w}^{(6) 0 j j} .
\end{aligned}
$$

For the shifts in the anomaly frequencies, the results are found to be [21]

$$
\begin{aligned}
& \delta \omega_{a}^{w}=2 \widetilde{b}_{w}^{3}-2 \widetilde{b}_{F, w}^{33} B, \\
& \delta \omega_{a}^{\bar{w}}=-2 \widetilde{b}_{w}^{* 3}+2 \widetilde{b}_{F, w}^{* 33} B,
\end{aligned}
$$

where various tilde and starred tilde coefficients are given by definitions (7), (11), (8), and (12). We note in passing that the shifts in the cyclotron and anomaly frequencies for an antifermion in results (20) and (22) reveals that they can be obtained from these for the 
fermion by changing the signs of all the basic coefficients for Lorentz violation that control CPT-odd effects, as might be expected.

Result (20) shows that the cyclotron frequency shifts due to Lorentz and CPT violation for a fermion are different from these for its corresponding antifermion. The same conclusion holds for the anomaly frequency shifts from the result (22). This implies that in the presence of Lorentz and CPT violation, the differences (15) and (17) do not vanish in general. For the charge-to-mass ratio comparisons, the result becomes

$$
\frac{(|q| / m)_{\bar{w}}}{(|q| / m)_{w}}-1 \longleftrightarrow \frac{\omega_{c}^{\bar{w}}}{\omega_{c}^{w}}-1=\frac{\delta \omega_{c}^{\bar{w}}-\delta \omega_{c}^{w}}{\omega_{c}^{w}},
$$

where the Lorentz- and CPT-invariant pieces in the measured cyclotron frequencies are exactly canceled by the CPT theorem if the same magnetic field is used. The notation $\longleftrightarrow$ indicates the correspondence between the experimental interpreted charge-to-mass ratio comparison and the measured frequency difference $\omega_{c}^{\bar{w}} / \omega_{c}^{w}-1$. For the $g$ factor comparison between a fermion and antifermion, the related ratio comes to be

$$
\frac{1}{2}\left(g_{w}-g_{\bar{w}}\right) \longleftrightarrow \frac{\omega_{a}^{w}}{\omega_{c}^{w}}-\frac{\omega_{a}^{\bar{w}}}{\omega_{c}^{\bar{w}}}=\frac{\delta \omega_{a}^{w}}{\omega_{c}^{w}}-\frac{\delta \omega_{a}^{\bar{w}}}{\omega_{c}^{\bar{w}}}
$$

where again all Lorentz- and CPT-invariant contributions are canceled out on the right side. We note that in the above expression (24), we only keep shifts in the anomaly frequencies $\delta \omega_{a}^{w}$ and $\delta \omega_{a}^{\bar{w}}$ due to Lorentz and CPT violation, as contributions from the cyclotron frequencies $\delta \omega_{c}^{w}$ and $\delta \omega_{c}^{\bar{w}}$ are suppressed by factors of $e B / m_{w}^{2}$, as evident from results (20) and (22). Even for a comparatively large magnetic field of $B \approx 5 \mathrm{~T}$ in a Penning trap, these factors are at orders of $e B / m_{e}^{2} \approx 10^{-9}$ for electrons or positrons, and $e B / m_{p}^{2} \approx 10^{-16}$ for protons or antiprotons, which can be ignored in Equation (24).

\subsection{Sidereal Variations}

The cyclotron and anomaly frequency shifts (20) and (22), which also appear in comparisons (23) and (24), are obtained in a particular apparatus frame $x^{a} \equiv\left(x^{1}, x^{2}, x^{3}\right)$, where the positive $\hat{x}_{3}$ axis is aligned with the applied magnetic field in the trap. However, as Earth rotates about its axis, this apparatus frame is not inertial. The standard canonical frame that is adopted in the literature to compare results from different experiments searching for Lorentz and CPT violations is called the Sun-centered frame $X^{J} \equiv(X, Y, Z)[36,37]$. In this frame, the $Z$ axis is defined to be aligned along the Earth's rotation axis, the $X$ axis points from the Earth to the Sun, and the $Y$ axis completes a right-handed coordinate system. The time origin of this coordinate system is chosen to be at the vernal equinox 2000. The coefficients for Lorentz violation in this frame are assumed to be constants in time and space $[4,8]$.

To explicitly express the relationship of the coefficients for Lorentz violation between the Sun-centered frame $X^{J} \equiv(X, Y, Z)$ and the apparatus frame $x^{a} \equiv\left(x^{1}, x^{2}, x^{3}\right)$, it is convenient to introduce a third frame called the standard laboratory frame $x^{j} \equiv(x, y, z)$, with the $z$ axis pointing to the local zenith, the $x$ axis aligned with the local south, and the $y$ axis completing a right-handed coordinate system. To relate the coordinates of these three frames, we define two rotation matrices, $R^{a j}$ and $R^{j J}$ [36,37], with $R^{a j}$ connecting $x^{j} \equiv(x, y, z)$ to $x^{a} \equiv\left(x^{1}, x^{2}, x^{3}\right)$ by $x^{a}=R^{a j} x^{j}$, and $R^{j J}$ relating $X^{J} \equiv(X, Y, Z)$ to $(x, y, z)$ by $x^{j}=R^{j J} X^{J}$. The expressions for these two rotation matrices are given by

$$
R^{j J}=\left(\begin{array}{ccc}
\cos \chi \cos \omega_{\oplus} T_{\oplus} & \cos \chi \sin \omega_{\oplus} T_{\oplus} & -\sin \chi \\
-\sin \omega_{\oplus} T_{\oplus} & \cos \omega_{\oplus} T_{\oplus} & 0 \\
\sin \chi \cos \omega_{\oplus} T_{\oplus} & \sin \chi \sin \omega_{\oplus} T_{\oplus} & \cos \chi
\end{array}\right),
$$




$$
R^{a j}=\left(\begin{array}{ccc}
\cos \gamma & \sin \gamma & 0 \\
-\sin \gamma & \cos \gamma & 0 \\
0 & 0 & 1
\end{array}\right) \times\left(\begin{array}{ccc}
\cos \beta & 0 & -\sin \beta \\
0 & 1 & 0 \\
\sin \beta & 0 & \cos \beta
\end{array}\right) \times\left(\begin{array}{ccc}
\cos \alpha & \sin \alpha & 0 \\
-\sin \alpha & \cos \alpha & 0 \\
0 & 0 & 1
\end{array}\right) \text {, }
$$

where $\omega_{\oplus} \approx 2 \pi /(23 \mathrm{~h} 56 \mathrm{~min})$ is the sidereal frequency of the Earth's rotation, $T_{\oplus}$ denotes the local sidereal time, $\chi$ specifies the colatitude of the laboratory, and $(\alpha, \beta, \gamma)$ are the Euler angles in the convenient " $y$-convention" of the rotation. The coordinates of the apparatus frame and the Sun-centered frame can then be related by using the following expression:

$$
x^{a}=R^{a j} x^{j}=R^{a j} R^{j J} x^{J} .
$$

The relationship between the coefficients for Lorentz violation in these two frames can also be derived from this result. We note that in the case that a vertical upward magnetic field is used in the trap, the Euler angles become $(\alpha, \beta, \gamma)=(0,0,0)$ and the rotation matrix $R^{a j}$ reduces to the identity matrix.

The above transformation (27) shows that in general the coefficients for Lorentz violation and thus the cyclotron and anomaly frequency shifts (20) and (22) determined in the apparatus frame depend on the sidereal time and the geometric location of the laboratory. As a result, signals observed in Earth-based experiments, including the above comparisons (23) and (24), can oscillate at harmonics of the Earth's sidereal frequency $\omega_{\oplus}$, with the amplitudes depending on the laboratory colatitude $\chi$. To explicitly illustrate this, here we consider a Penning-trap experiment located at colatitude $\chi$ and assume the magnetic field is aligned with the $z$ axis. We give two explicit examples of the transformation of coefficients for Lorentz violation using Equation (27). We first focus on the single-index laboratory-frame coefficient $\widetilde{b}_{w}^{\prime 3}$, which appears in the shifts of the cyclotron frequency (20). Applying the transformation (27) and taking $(\alpha, \beta, \gamma)=(0,0,0)$ imply

$$
\widetilde{b}_{w}^{\prime 3}=\cos \omega_{\oplus} T_{\oplus} \widetilde{b}_{w}^{\prime X} \sin \chi+\sin \omega_{\oplus} T_{\oplus} \widetilde{b}_{w}^{\prime Y} \sin \chi+\widetilde{b}_{w}^{\prime Z} \cos \chi .
$$

This result relates the tilde coefficients $\widetilde{b}_{w}^{\prime 3}$ observed in the noninertial apparatus frame to the constant coefficients $\widetilde{b}_{w}^{J}$ with $J=X, Y, Z$ in the canonical inertial Sun-centered frame. The expression contains terms proportional to the first harmonic in the Earth's sidereal frequency and a constant. The colatitude dependence is evident from the factors $\sin \chi$ and $\cos \chi$ appearing above.

A slightly more complicated result can also be obtained for the sum of the two-index tilde quantities $\widetilde{c}_{w}^{11}+\widetilde{c}_{w}^{22}$ in an analogous fashion. Applying the rotation (27) for each index with $R^{a j}$ being the identity matrix yields

$$
\begin{aligned}
\widetilde{c}_{w}^{11}+\widetilde{c}_{w}^{22}= & \cos 2 \omega_{\oplus} T_{\oplus}\left(-\frac{1}{2}\left(\widetilde{c}_{w}^{X X}-\widetilde{c}_{w}^{Y Y}\right) \sin ^{2} \chi\right)+\sin 2 \omega_{\oplus} T_{\oplus}\left(-\widetilde{c}_{w}^{(X Y)} \sin ^{2} \chi\right) \\
& +\cos \omega_{\oplus} T_{\oplus}\left(-\widetilde{c}_{w}^{(X Z)} \sin 2 \chi\right)+\sin \omega_{\oplus} T_{\oplus}\left(-\widetilde{c}_{w}^{(Y Z)} \sin 2 \chi\right) \\
& +\frac{1}{4}\left(\widetilde{c}_{w}^{X X}+\widetilde{c}_{w}^{Y Y}\right)(3+\cos 2 \chi)+\widetilde{c}_{w}^{Z Z} \sin ^{2} \chi
\end{aligned}
$$

where a parenthes on two indices of the coefficients indicates symmetrization with a factor of 1/2. For example, $\widetilde{c}_{w}^{(X Y)}=\left(\widetilde{c}_{w}^{X Y}+\widetilde{c}_{w}^{Y X}\right) / 2$. It is revealed from the result (29) that the sum of the tilde coefficients $\widetilde{c}_{w}^{11}+\widetilde{c}_{w}^{22}$ in the apparatus frame depends on six independent quantities $\widetilde{c}_{w}^{(J K)}$ with $J, K=X, Y, Z$ in the Sun-centered frame, producing up to second harmonics in the sidereal frequency of the Earth's rotation. For both examples (28) and (29), if the applied magnetic field points along a generic direction, trigonometric functions of the Euler angles $(\alpha, \beta, \gamma)$ appear as well.

At the end of this subsection, we note that the revolution of the Earth about the Sun can generate additional types of time variations for the coefficients for Lorentz violation, such as the boost of the Earth relative to the $\operatorname{Sun} \beta_{\oplus} \approx 10^{-4}$, and the boost of the laboratory 
due to the Earth's rotation $\beta_{L} \approx 10^{-6}$. However, as studied in the literature $[27,28,38-40]$, such boost effects are suppressed by one or more powers of their boost factors $\beta_{\oplus}$ and $\beta_{L}$ compared to these from rotations. Therefore, we treat them as negligible effects in the present work.

\section{Experiments}

In this section, we analyze existing Penning-trap experiments that compare the chargeto-mass ratios and the $g$ factors between protons, antiprotons, electrons, and positrons. We use reported results for the comparisons (23) and (24) from these experiments to constrain the relevant Sun-centered frame tilde coefficients for Lorentz violation.

\subsection{The Charge-to-Mass Ratios}

For charge-to-mass ratio comparisons between a particle and its corresponding antiparticle, the result (23) implies that the relevant tilde coefficients for Lorentz violation are $\widetilde{b}_{w}^{\prime 3}, \widetilde{c}_{w}^{11}+\widetilde{c}_{w}^{22}, \widetilde{b}_{w}^{311}+\widetilde{b}_{w}^{322}, \widetilde{b}_{w}^{\prime * 3}, \widetilde{c}_{w}^{* 11}+\widetilde{c}_{w}^{* 22}$, and $\widetilde{b}_{w}^{* 311}+\widetilde{b}_{w}^{* 322}$ in the apparatus frame, given in the result (20). The transformations of these tilde coefficients into the Sun-centered frame depend on the field configuration in the trap. For a typical Penning-trap experiment, the magnetic field is oriented either horizontally or vertically. The work in [22] presents a complete list of transformation results for these tilde coefficients for the above two field orientations. Here, for completeness, we include them in Appendix A. From these transformation results, it shows that for a given fermion of species $w$, the relevant quantities in the Sun-centered frame that are related to the charge-to-mass comparisons in a Penning trap are the following 54 independent tilde coefficients, $\widetilde{b}_{w}^{\prime}, \widetilde{c}_{w}^{(J K)}, \widetilde{b}_{w}^{J(K L)}, \widetilde{b}_{w}^{\prime * J}, \widetilde{c}_{w}^{*(J K)}$, and $\widetilde{b}_{w}^{* J(K L)}$. In the following subsections, we apply the reported precisions for the charge-to-mass ratio comparisons from Penning-trap experiments to set bounds on the relevant tilde coefficients for Lorentz violation.

\subsubsection{The Proton Sector}

We start the analysis with the charge-to-mass comparisons between protons and antiprotons. In a Penning-trap experiment located at CERN by the ATRAP collaboration, Gabrielse and his group achieved a precision of $90 \mathrm{ppt}$ for the proton-antiproton charge-to-mass ratio comparison [41]. The experiment used a trap with a vertical uniform magnetic field $B=5.85 \mathrm{~T}$. Recently, another Penning-trap experiment at CERN by the BASE collaboration led by Ulmer improved the comparison to the record sensitivity of 69 ppt [42], by applying a horizontal magnetic field $B=1.946 \mathrm{~T}$ which is oriented $60^{\circ}$ east of north. For the measurement of the charge-to-mass ratio of a proton, both experiments used a trapped hydrogen ion $\left(\mathrm{H}^{-}\right)$as a proxy for the proton to eliminate systematic shifts caused by polarity switching of the trapping voltages. The charge-to-mass ratio comparison between an antiproton and a proton is then related to that between an antiproton and a hydrogen ion by

$$
\frac{(|q| / m)_{\bar{p}}}{(|q| / m)_{p}}-1=\frac{(|q| / m)_{\bar{p}}}{R(|q| / m)_{\mathrm{H}^{-}}}-1 \longleftrightarrow \frac{\delta \omega_{c}^{\bar{p}}-R \delta \omega_{c}^{\mathrm{H}^{-}}}{R \omega_{c}^{\mathrm{H}^{-}}}
$$

where $R=m_{\mathrm{H}^{-}} / m_{p}=1.001089218754$ is the ratio of the mass between a hydrogen ion and a proton [42], and $\omega_{c}^{\mathrm{H}^{-}}$is the cyclotron frequency for the hydrogen ion, with $\delta \omega_{c}^{\mathrm{H}^{-}}$being the corresponding shifts. To obtain $\delta \omega_{c}^{\mathrm{H}^{-}}$, one can take $w=\mathrm{H}^{-}$in the expression (20) and the related tilde coefficients for Lorentz violation become the effective ones for a hydrogen ion. Expressing these effective coefficients in terms of the corresponding fundamental coefficients for the hydrogen ion constituents, which are the coefficients for electrons and protons, is challenging due to nonperturbative issues including binding effects in the composite hydrogen ion. However, an approximation to these coefficient relations can be obtained by treating the wave function of the hydrogen ion as a product of the wave functions of a proton and two electrons. Applying perturbation theory at the lowest order 
and ignoring the related binding energies, the cyclotron frequency shifts $\delta \omega_{c}^{\mathrm{H}^{-}}$of the hydrogen ion due to Lorentz and CPT violation can then be approximated as the sum of these for its constituents, $\delta \omega_{c}^{\mathrm{H}^{-}} \approx \delta \omega_{c}^{p}+2 \delta \omega_{c}^{e^{-}}$. Substituting this into the result (30) yields

$$
\frac{(|q| / m)_{\bar{p}}}{(|q| / m)_{p}}-1 \longleftrightarrow \frac{\delta \omega_{c}^{\bar{p}}-R \delta \omega_{c}^{p}-2 R \delta \omega_{c}^{e^{-}}}{R \omega_{c}^{\mathrm{H}^{-}}} .
$$

As shown from the above result, the choice of using a hydrogen ion as a proxy for the proton in the Penning trap provides sensitivities not only to the coefficients for Lorentz violation in the proton sector, but also introduces additional sensitivities to these for electrons. Putting the above discussion together, the related Sun-centered frame tilde coefficients for Lorentz violation that are sensitive to Penning-trap experiments comparing the chargeto-mass ratios between protons and antiprotons are the following 81 independent tilde quantities: $\widetilde{b}_{p}^{\prime J}, \widetilde{c}_{p}^{(J K)}, \widetilde{b}_{p}^{J(K L)}, \widetilde{b}_{p}^{\prime * J}, \widetilde{c}_{p}^{*(J K)}, \widetilde{b}_{p}^{* J(K L)}, \widetilde{b}_{e}^{\prime J}, \widetilde{c}_{e}^{(J K)}$, and $\widetilde{b}_{e}^{J(K L)}$. The published results for the comparison (23) from both the ATRAP and the BASE experiments can be adopted to set bounds on these tilde coefficients for Lorentz violation.

For the ATRAP experiment, the reported precision was obtained by analyzing the measurements of the cyclotron frequencies in a time-averaged way, so any oscillating effects in the difference (31) averaged out. This implies that only the constant terms that appear in the transformation results in the first half of Appendix A can be constrained using the published precision. Applying expression (31) by taking the reported precision of $90 \mathrm{ppt}$ for $(|q| / m)_{\bar{p}} /(|q| / m)_{p}-1$ and identifying $\omega_{c}^{\mathrm{H}^{-}}=2 \pi \times 89.3 \mathrm{MHz}$ given in the ATRAP experiment, the following limit can be obtained:

$$
\left|\delta \omega_{c}^{\bar{p}}-1.001 \delta \omega_{c}^{p}-2.002 \delta \omega_{c}^{e^{-}}\right|_{\text {const }} \lesssim 3.33 \times 10^{-26} \mathrm{GeV},
$$

where the subscript "const" indicates that only the constant terms in the transformations results are relevant to the limit. However, future sidereal-variation analysis of the measurements of the cyclotron frequencies can provide constraints on the non-constant terms that appear in the harmonics in the transformation results.

For the experiment carried out by the BASE collaboration, as the magnetic field was oriented at $60^{\circ}$ east of north, both of the transformation matrices (25) and (26) are required to relate the tilde coefficients for Lorentz violation in the apparatus frame to these in the Sun-centered frame. For a general horizontal magnetic field with an angle $\theta$ measured from the local south in the counterclockwise direction, the corresponding Euler angles are found to be $(\alpha, \beta, \gamma)=(\theta, \pi / 2,0)$. The second half of Appendix A lists the full expressions of the transformations for the related tilde coefficients for Lorentz violation. The BASE experiment analyzed the data of the charge-to-mass ratio comparisons to search for both time-averaged effects and sidereal variations in the first harmonics of the Earth's rotation frequency. Therefore, the reported results can be taken to set bounds on not only the constant terms but also on the terms proportional to the first harmonics in the transformation results given in Appendix A. Using the reported $69 \mathrm{ppt}$ for the time-averaged precision and $720 \mathrm{ppt}$ for the limit of the first harmonic amplitude for the comparison (31), and taking $\omega_{c}^{\mathrm{H}^{-}}=2 \pi \times 29.635 \mathrm{MHz}$ for the BASE experiment, the following limits are obtained:

$$
\left|\delta \omega_{c}^{\bar{p}}-1.001 \delta \omega_{c}^{p}-2.002 \delta \omega_{c}^{e^{-}}\right|_{\text {const }} \lesssim 8.46 \times 10^{-27} \mathrm{GeV}
$$

and

$$
\left|\delta \omega_{c}^{\bar{p}}-1.001 \delta \omega_{c}^{p}-2.002 \delta \omega_{c}^{e^{-}}\right|_{1 \mathrm{st}} \lesssim 8.83 \times 10^{-26} \mathrm{GeV},
$$

where the subscript "const" in the limit (33) has similar meaning as the one in (32), while the subscript "1st" in the limit (34) specifies only the amplitude of the first harmonics in the sidereal variation.

The limit (32) for the ATRAP experiment and limits (33) and (34) for the BASE experiment set constraints on a combination of the Sun-centered frame tilde coefficients $\widetilde{b}_{p}^{\prime}$, 
$\widetilde{c}_{p}^{(J K)}, \widetilde{b}_{p}^{J(K L)}, \widetilde{b}_{p}^{\prime * J}, \widetilde{c}_{p}^{*(J K)}, \widetilde{b}_{p}^{* J(K L)}, \widetilde{b}_{e}^{\prime J}, \widetilde{c}_{e}^{(J K)}$, and $\widetilde{b}_{e}^{J(K L)}$. These constraints can be obtained by substituting result (20) in limit (32) and applying the corresponding transformations given in Appendix A with $\chi=43.8^{\circ}$ for the ATRAP experiment, and substituting result (20) in both limits (33) and (34) and identifying $\theta=2 \pi / 3$ and $\chi=43.8^{\circ}$ in the transformation expressions in Appendix A for the BASE experiment. To get some intuition about the scope of the constraints on the individual component of the tilde coefficients, we can take a common practice that is adopted in many subfields searching for Lorentz and CPT violation [7], which assumes that only one individual tilde coefficient is nonzero at a time. From the ATRAP limit (32), constraints on 27 independent tilde coefficients for Lorentz violation are obtained, while from the BASE limits (33) and (34), a total of 69 independent tilde coefficients for Lorentz violation are constrained. In Tables 1 and 2, we summarize the constraints in the proton sector and the electron sector, respectively. In both tables, the first column lists the individual components, the second column presents the corresponding constraint on the modulus of the component, the third column specifies the related experiment, and the related reference is given in the final column. We note that some of the tilde coefficients for Lorentz violation are constrained by both the ATRAP and the BASE experiments. To keep the results clean, we only keep the more stringent ones in both tables.

Table 1. Constraints on tilde coefficients for Lorentz violation in the proton sector using the chargeto-mass ratio comparisons from the ATRAP and the BASE experiments.

\begin{tabular}{|c|c|c|c|}
\hline Coefficient & Constraint & Experiment & Reference \\
\hline$\left|\widetilde{b}_{p}^{\prime Z}\right|,\left|\widetilde{b}_{p}^{\prime * Z}\right|$ & $<1 \times 10^{-10} \mathrm{GeV}$ & ATRAP & [41] \\
\hline$\left|\widetilde{c}_{p}^{X X}\right|,\left|\widetilde{c}_{p}^{* X X}\right|$ & $<1 \times 10^{-10}$ & ATRAP & [41] \\
\hline$\left|\widetilde{c}_{p}^{Y Y}\right|,\left|\widetilde{c}_{p}^{* Y Y}\right|$ & $<1 \times 10^{-10}$ & ATRAP & [41] \\
\hline$\left|\widetilde{c}_{p}^{Z Z}\right|,\left|\vec{c}_{p}^{* Z Z}\right|$ & $<8 \times 10^{-11}$ & BASE & [42] \\
\hline$\left|\widetilde{b}_{p}^{X(X Z)}\right|,\left|\widetilde{b}_{p}^{* X(X Z)}\right|$ & $<2 \times 10^{-10} \mathrm{GeV}^{-1}$ & BASE & [42] \\
\hline$\left|\widetilde{b}_{p}^{Y(Y Z)}\right|,\left|\widetilde{b}_{p}^{* Y(Y Z)}\right|$ & $<2 \times 10^{-10} \mathrm{GeV}^{-1}$ & BASE & [42] \\
\hline$\left|\widetilde{b}_{p}^{Z Z Z}\right|,\left|\widetilde{b}_{p}^{* Z Z Z}\right|$ & $<2 \times 10^{-10} \mathrm{GeV}^{-1}$ & BASE & [42] \\
\hline$\left|\widetilde{b}_{p}^{Z X X}\right|,\left|\widetilde{b}_{p}^{* Z X X}\right|$ & $<2 \times 10^{-10} \mathrm{GeV}^{-1}$ & ATRAP & [41] \\
\hline$\left|\widetilde{b}_{p}^{Z Y Y}\right|,\left|\widetilde{b}_{p}^{* Z Y Y}\right|$ & $<2 \times 10^{-10} \mathrm{GeV}^{-1}$ & ATRAP & [41] \\
\hline$\left|\widetilde{b}_{p}^{\prime X}\right|,\left|\widetilde{b}_{p}^{\prime * X}\right|$ & $<7 \times 10^{-10} \mathrm{GeV}$ & BASE & [42] \\
\hline$\left|\widetilde{b}_{p}^{\prime Y}\right|,\left|\widetilde{b}_{p}^{\prime * Y}\right|$ & $<7 \times 10^{-10} \mathrm{GeV}$ & BASE & [42] \\
\hline$\left|\widetilde{c}_{p}^{(X Z)}\right|,\left|\widetilde{c}_{p}^{*(X Z)}\right|$ & $<1 \times 10^{-9}$ & BASE & [42] \\
\hline$\left|\widetilde{c}_{p}^{(Y Z)}\right|,\left|\widetilde{c}_{p}^{*(Y Z)}\right|$ & $<1 \times 10^{-9}$ & BASE & [42] \\
\hline$\left|\widetilde{b}_{p}^{X X X}\right|,\left|\widetilde{b}_{p}^{* X X X}\right|$ & $<2 \times 10^{-9} \mathrm{GeV}^{-1}$ & BASE & [42] \\
\hline$\left|\widetilde{b}_{p}^{X(X Y)}\right|,\left|\widetilde{b}_{p}^{* X(X Y)}\right|$ & $<2 \times 10^{-9} \mathrm{GeV}^{-1}$ & BASE & [42] \\
\hline$\left|\widetilde{b}_{p}^{X Y Y}\right|,\left|\widetilde{b}_{p}^{* X Y Y}\right|$ & $<1 \times 10^{-9} \mathrm{GeV}^{-1}$ & BASE & [42] \\
\hline$\left|\widetilde{b}_{p}^{X Z Z}\right|,\left|\widetilde{b}_{p}^{* X Z Z}\right|$ & $<9 \times 10^{-10} \mathrm{GeV}^{-1}$ & BASE & [42] \\
\hline$\left|\widetilde{b}_{p}^{Y X X}\right|,\left|\widetilde{b}_{p}^{* Y X X}\right|$ & $<1 \times 10^{-9} \mathrm{GeV}^{-1}$ & BASE & [42] \\
\hline$\left|\widetilde{b}_{p}^{Y(X Y)}\right|, \widetilde{b}_{p}^{* Y(X Y)} \mid$ & $<2 \times 10^{-9} \mathrm{GeV}^{-1}$ & BASE & [42] \\
\hline$\left|\widetilde{b}_{p}^{Y Y Y}\right|,\left|\widetilde{b}_{p}^{* Y Y Y}\right|$ & $<2 \times 10^{-9} \mathrm{GeV}^{-1}$ & BASE & [42] \\
\hline$\left|\widetilde{b}_{p}^{Y Z Z}\right|,\left|\widetilde{b}_{p}^{* Y Z Z}\right|$ & $<9 \times 10^{-10} \mathrm{GeV}^{-1}$ & BASE & [42] \\
\hline$\left|\widetilde{b}_{p}^{Z(X Z)}\right|,\left|\widetilde{b}_{p}^{* Z(X Z)}\right|$ & $<3 \times 10^{-9} \mathrm{GeV}^{-1}$ & BASE & [42] \\
\hline$\left|\widetilde{b}_{p}^{Z(Y Z)}\right|,\left|\widetilde{b}_{p}^{* Z(Y Z)}\right|$ & $<3 \times 10^{-9} \mathrm{GeV}^{-1}$ & BASE & [42] \\
\hline
\end{tabular}


Table 2. Constraints on tilde coefficients for Lorentz violation in the electron sector using the charge-to-mass ratio comparisons from the ATRAP and the BASE experiments.

\begin{tabular}{lllc}
\hline Coefficient & Constraint & Experiment & Reference \\
\hline$\left|\widetilde{b}_{e}^{\prime Z}\right|$ & $<2 \times 10^{-17} \mathrm{GeV}$ & ATRAP & {$[41]$} \\
$\left|\widetilde{c}_{e}^{X X}\right|$ & $<3 \times 10^{-14}$ & ATRAP & {$[41]$} \\
$\left|\widetilde{c}_{e}^{Y Y}\right|$ & $<3 \times 10^{-14}$ & ATRAP & {$[41]$} \\
$\left|\widetilde{c}_{e}^{Z Z}\right|$ & $<2 \times 10^{-14}$ & BASE & {$[42]$} \\
$\left|\widetilde{b}_{e}^{X(X Z)}\right|$ & $<1 \times 10^{-10} \mathrm{GeV}^{-1}$ & BASE & {$[42]$} \\
$\left|\widetilde{b}_{e}^{Y(Y Z)}\right|$ & $<1 \times 10^{-10} \mathrm{GeV}^{-1}$ & BASE & {$[42]$} \\
$\left|\widetilde{b}_{e}^{Z Z Z}\right|$ & $<1 \times 10^{-10} \mathrm{GeV}^{-1}$ & BASE & {$[42]$} \\
$\left|\widetilde{b}_{e}^{Z X X}\right|$ & $<9 \times 10^{-11} \mathrm{GeV}^{-1}$ & ATRAP & {$[41]$} \\
$\left|\widetilde{b}_{e}^{Z Y Y}\right|$ & $<9 \times 10^{-11} \mathrm{GeV}^{-1}$ & ATRAP & {$[41]$} \\
$\left|\widetilde{b}_{e}^{\prime X}\right|$ & $<1 \times 10^{-16} \mathrm{GeV}^{\prime X}$ & BASE & {$[42]$} \\
$\left|\widetilde{b}_{e}^{\prime Y}\right|$ & $<1 \times 10^{-16} \mathrm{GeV}^{\prime Y}$ & BASE & {$[42]$} \\
$\left|\widetilde{c}_{e}^{X Z)}\right|$ & $<3 \times 10^{-13}$ & BASE & {$[42]$} \\
$\left|\widetilde{c}_{e}^{(Y Z)}\right|$ & $<3 \times 10^{-13}$ & BASE & {$[42]$} \\
$\left|\widetilde{b}_{e}^{X X X}\right|$ & $<1 \times 10^{-9} \mathrm{GeV}^{-1}$ & BASE & {$[42]$} \\
$\left|\widetilde{b}_{e}^{X(X Y)}\right|$ & $<9 \times 10^{-10} \mathrm{GeV}^{-1}$ & BASE & {$[42]$} \\
$\left|\widetilde{b}_{e}^{X Y Y}\right|$ & $<5 \times 10^{-10} \mathrm{GeV}^{-1}$ & BASE & {$[42]$} \\
$\left|\widetilde{b}_{e}^{X Z Z}\right|$ & $<5 \times 10^{-10} \mathrm{GeV}^{-1}$ & BASE & {$[42]$} \\
$\left|\widetilde{b}_{e}^{Y X X}\right|$ & $<5 \times 10^{-10} \mathrm{GeV}^{-1}$ & BASE & {$[42]$} \\
$\left|\widetilde{b}_{e}^{Y(X Y)}\right|$ & $<9 \times 10^{-10} \mathrm{GeV}^{-1}$ & BASE & {$[42]$} \\
$\left|\widetilde{b}_{e}^{Y Y Y}\right|$ & $<1 \times 10^{-9} \mathrm{GeV}^{-1}$ & BASE & {$[42]$} \\
$\left|\widetilde{b}_{e}^{Y Z Z}\right|$ & $<5 \times 10^{-10} \mathrm{GeV}^{-1}$ & BASE & {$[42]$} \\
$\left|\widetilde{b}_{e}^{Z(X Z)}\right|$ & $<2 \times 10^{-9} \mathrm{GeV}^{-1}$ & BASE & {$[42]$} \\
$\left|\widetilde{b}_{e}^{Z(Y Z)}\right|$ & $<2 \times 10^{-9} \mathrm{GeV}^{-1}$ & BASE & {$[42]$} \\
\hline & & & \\
\hline
\end{tabular}

\subsubsection{The Electron Sector}

For the comparison of the charge-to-mass ratios between an electron and a positron, the current most accurate result was made in an experiment at the University of Washington, with a precision at $130 \mathrm{ppb}$ [43]. From the comparison (23), this time-average result gives the following limit:

$$
\left|\delta \omega_{c}^{e^{-}}-\delta \omega_{c}^{e^{+}}\right|_{\text {const }} \lesssim 7.66 \times 10^{-20} \mathrm{GeV} .
$$

Following a similar analysis as the one used for the proton sector in the previous subsection, one can use the colatitude and magnetic field that are relevant to this experiment $\chi=42.5^{\circ}$ and $B=5.1 \mathrm{~T}$ upward, together with the transformation expressions given in Appendix A, to obtain the constraints on the tilde coefficients for Lorentz violation $\widetilde{b}_{e}^{\prime J}, \widetilde{c}_{e}^{(J K)}$, $\widetilde{b}_{e}^{J(K L)}, \widetilde{b}_{e}^{\prime * J}, \widetilde{c}_{e}^{*(J K)}$, and $\widetilde{b}_{e}^{* J(K L)}$. We summarize the results in Table 3, which is organized in the same way as Tables 1 and 2. We note that some of the constraints of the tilde coefficients from the electron-positron charge-to-mass ratio comparison are not comparable to these given in Table 2 from the proton-antiproton comparison, so we do not include them in Table 3. We also note that the Penning trap experiment at the University of Washington used a radioactive source of positrons that requires special precautions. Efforts in applying a safe source and ensuring efficient positron accumulation are currently being made at 
both Harvard University and Northwestern University [44,45], providing great potential for improving the current bounds for the tilde coefficients listed in Tables 2 and 3.

Table 3. Constraints on tilde coefficients for Lorentz violation in the electron sector using the charge-to-mass ratio comparisons from the experiment at the University of Washington.

\begin{tabular}{lllc}
\hline Coefficient & Constraint & Experiment & Reference \\
\hline$\left|\widetilde{b}_{e}^{\prime * Z}\right|$ & $<9 \times 10^{-11} \mathrm{GeV}$ & Washington & {$[43]$} \\
$\left|\widetilde{c}_{e}^{* X X}\right|$ & $<2 \times 10^{-7}$ & Washington & {$[43]$} \\
$\left|\widetilde{c}_{e}^{* Y Y}\right|$ & $<2 \times 10^{-7}$ & Washington & {$[43]$} \\
$\left|\widetilde{c}_{e}^{* Z Z}\right|$ & $<3 \times 10^{-7}$ & Washington & {$[43]$} \\
$\left|\widetilde{b}_{e}^{* X(X Z)}\right|$ & $<8 \times 10^{-4} \mathrm{GeV}^{-1}$ & Washington & {$[43]$} \\
$\left|\widetilde{b}_{e}^{* Y(Y Z)}\right|$ & $<8 \times 10^{-4} \mathrm{GeV}^{-1}$ & Washington & {$[43]$} \\
$\mid \widetilde{b}_{e}^{* Z Z Z \mid}$ & $<8 \times 10^{-4} \mathrm{GeV}^{-1}$ & Washington & {$[43]$} \\
$\left|\widetilde{b}_{e}^{* Z X X}\right|$ & $<4 \times 10^{-4} \mathrm{GeV}^{-1}$ & Washington & {$[43]$} \\
$\left|\widetilde{b}_{e}^{* Z Y Y}\right|$ & $<4 \times 10^{-4} \mathrm{GeV}^{-1}$ & Washington & {$[43]$} \\
\hline
\end{tabular}

\subsection{The $g$ Factors and Magnetic Moments}

For the $g$ factor and magnetic moment comparisons between particles and antiparticles using Penning traps, the result (24) implies that the relevant coefficients for Lorentz violation in the apparatus frame are these given in Equation (22), $\widetilde{b}_{w}^{3}, \widetilde{b}_{w}^{* 3}, \widetilde{b}_{F, w^{\prime}}^{33}$ and $\widetilde{b}_{F, w}^{* 33}$ For a Penning trap with a vertical or horizontal magnetic field, the expressions of the transformation for these tilde coefficients into the Sun-centered frame are also given in Appendix A. The results show that there are 18 independent components of the tilde coefficients for each fermion species $w$, given by $\widetilde{b}_{w}^{J}, \widetilde{b}_{w}^{* J}, \widetilde{b}_{F, w}^{(J K)}$, and $\widetilde{b}_{F, w}^{*(J K)}$. In the subsection follows, we list the related Penning-trap experiments and use the reported comparison results to set bounds on the corresponding tilde coefficients for Lorentz violation.

\subsubsection{The Proton Sector}

In the proton sector, the current best measurements of the magnetic moments for both a proton and an antiproton were achieved by the BASE collaboration. The proton magnetic moment measurement has a sensitivity of $0.3 \mathrm{ppb}$ using a Penning trap located at Mainz [46], improving their previous best measurement [47] by a factor of 11 . The antiproton magnetic moment measurement was measured to a precision of $1.5 \mathrm{ppb}$ with a similar Penning trap located at CERN [48]. Combining the reported $0.3 \mathrm{ppb}$ and $1.5 \mathrm{ppb}$ precisions for the time-averaged measurements, and identifying $\omega_{c}^{p}=2 \pi \times 28.96 \mathrm{MHz}$ and $\omega_{c}^{\bar{p}}=2 \pi \times 29.66 \mathrm{MHz}$ for each experiment, comparison (24) yields

$$
\left|\delta \omega_{a}^{p}-0.98 \delta \omega_{a}^{\bar{p}}\right|_{\text {const }} \lesssim 9.53 \times 10^{-25} \mathrm{GeV},
$$

where same subscript "const" is used to specify only the constant terms in the transformation are relevant to the above limit.

The corresponding constraints for the combinations of the tilde coefficients in the Suncentered frame can be obtained by applying the corresponding transformation results given in Appendix A that are related to the specific field configuration in the trap and substituting the numerical values of the laboratory quantities for both experiments in limit (36). For the proton magnetic moment measurement at Mainz, the colatitude is $\chi=40.0^{\circ}$ and the magnetic field $B=1.9 \mathrm{~T}$ points $\theta=18^{\circ}$ from local south in the counterclockwise direction [46]. For the experiment measuring the antiproton magnetic moment at CERN, the trap is located at a slight different colatitude $\chi=43.8^{\circ}$ and the magnetic field $B=1.95 \mathrm{~T}$ 
points $\theta=120^{\circ}$ in the same convention as above [48]. Adopting the same assumption that only one tilde coefficient is nonzero at a time, the constraint on each independent tilde coefficient can be obtained. We summarize them in Table 4 in the same fashion as before.

Table 4. Constraints on tilde coefficients for Lorentz violation in the proton sector using the $g$ factor comparison from the BASE experiments at Mainz and CERN.

\begin{tabular}{llcc}
\hline Coefficient & Constraint & Experiment & Reference \\
\hline$\left|\widetilde{b}_{p}^{Z}\right|$ & $<8 \times 10^{-25} \mathrm{GeV}$ & BASE & {$[46,48]$} \\
$\left|\widetilde{b}_{p}^{* Z}\right|$ & $<1 \times 10^{-24} \mathrm{GeV}$ & BASE & {$[46,48]$} \\
$\left|\widetilde{b}_{F, p}^{X X}+\widetilde{b}_{F, p}^{Y Y}\right|$ & $<4 \times 10^{-9} \mathrm{GeV}^{-1}$ & BASE & {$[46,48]$} \\
$\left|\widetilde{b}_{F, p}^{Z Z}\right|$ & $<3 \times 10^{-9} \mathrm{GeV}^{-1}$ & BASE & {$[46,48]$} \\
$\left|\widetilde{b}_{F, p}^{* X X}+\widetilde{b}_{F, p}^{* Y Y}\right|$ & $<3 \times 10^{-9} \mathrm{GeV}^{-1}$ & BASE & {$[46,48]$} \\
$\left|\widetilde{b}_{F, p}^{* Z Z}\right|$ & $<1 \times 10^{-8} \mathrm{GeV}^{-1}$ & BASE & {$[46,48]$} \\
\hline
\end{tabular}

At the end of this subsection, we point out that a sidereal-variation analysis of the anomaly frequencies for both protons and antiprotons is currently being carried out by the BASE collaboration. This could in principle set bounds on other components of the tilde coefficients $\widetilde{b}_{p}^{J}, \widetilde{b}_{F, p}^{(J K)}, \widetilde{b}_{p}^{* I}$, and $\widetilde{b}_{F, p}^{*(J K)}$ that have not been constrained before. The BASE collaboration is also developing a quantum logic readout system to allow more rapid measurements of the anomaly frequencies in the trap [49], which will offer excellent opportunities to perform a sidereal-variation analysis of the experimental data, with great potential to set more stringent limits on the tilde coefficients for Lorentz violation.

\subsubsection{The Electron Sector}

In the electron sector, the comparison of the anomaly frequencies between electrons and positrons were performed in a Penning-trap experiment at the University of Washington, with a precision of about $2 \mathrm{ppt}$ [50]. The experimental data were analyzed in a time-averaged way to obtain a constraint of $b \lesssim 50 \mathrm{rad} / \mathrm{s}$ using the notation given in [50]. Translating to the notation in this work yields

$$
\left|\delta \omega_{a}^{e^{-}}-\delta \omega_{a}^{e^{+}}\right|_{\text {const }} \lesssim 2.09 \times 10^{-23} \mathrm{GeV} .
$$

Taking the experimental quantities $\chi=42.5^{\circ}$ and $B=5.85 \mathrm{~T}$ upward, as well as the transformation presented in Appendix A, the limit (37) can be converted to constraints on the following Sun-centered frame tilde coefficients, $\widetilde{b}_{e}^{Z}, \widetilde{b}_{e}^{* Z}, \widetilde{b}_{F, e}^{X X}+\widetilde{b}_{F, e}^{Y Y}, \widetilde{b}_{F, e}^{* X X}+\widetilde{b}_{F, e}^{* Y Y}, \widetilde{b}_{F, e}^{Z Z}$, and $\widetilde{b}_{F, e}^{* Z Z}$.

The measurement of the $g$ factor for electrons has reached a record precision of 0.28 ppt at Harvard University [1]. A sidereal-variation analysis of the anomaly frequencies was performed at the frequencies of $\omega_{\oplus}$ and $2 \omega_{\oplus}$, yielding the same limit on the amplitudes of both the first and the second harmonics in the oscillation, $\left|\delta \omega_{a}^{e}\right|_{1 \text { st } / 2 \mathrm{nd}} \lesssim 2 \pi \times 0.05 \mathrm{~Hz}$ [24]. Converting the results in natural units gives the following limits:

$$
\left|\delta \omega_{a}^{e^{-}}\right|_{1 \text { st }} \lesssim 2.07 \times 10^{-25} \mathrm{GeV},
$$

and

$$
\left|\delta \omega_{a}^{e^{-}}\right|_{\text {2nd }} \lesssim 2.07 \times 10^{-25} \mathrm{GeV} .
$$

Taking the magnetic field adopted in the experiment as $B=5.36 \mathrm{~T}$ in the local upward direction with the geometrical colatitude $\chi=47.6^{\circ}$ and applying the transformation results in Appendix A, constraints on the following additional components of the tilde coefficients, 
$\widetilde{b}_{e}^{X}, \widetilde{b}_{e}^{Y}, \widetilde{b}_{F, e}^{(X Y)}, \widetilde{b}_{F, e}^{(X Z)}, \widetilde{b}_{F, e}^{(Y Z)}$, and $\widetilde{b}_{F, e}^{X X}-\widetilde{b}_{F, e}^{Y Y}$ are obtained. The results from the University of Washington and Harvard University are summarized in Table 5.

Table 5. Constraints on tilde coefficients for Lorentz violation in the electron sector using the $g$ factor measurements from the experiments at the University of Washington and Harvard University.

\begin{tabular}{llcc}
\hline Coefficient & Constraint & Experiment & Reference \\
\hline$\left|\widetilde{b}_{e}^{X}\right|$ & $<1 \times 10^{-25} \mathrm{GeV}$ & Harvard & {$[24]$} \\
$\left|\widetilde{b}_{e}^{Y}\right|$ & $<1 \times 10^{-25} \mathrm{GeV}$ & Harvard & {$[24]$} \\
$\left|\widetilde{b}_{e}^{Z}\right|$ & $<7 \times 10^{-24} \mathrm{GeV}$ & Washington & {$[50]$} \\
$\left|\widetilde{b}_{e}^{* Z}\right|$ & $<7 \times 10^{-24} \mathrm{GeV}$ & Washington & {$[50]$} \\
$\left|\widetilde{b}_{F, e}^{X X}+\widetilde{b}_{F, e}^{Y Y}\right|$ & $<2 \times 10^{-8} \mathrm{GeV}^{-1}$ & Washington & {$[50]$} \\
$\left|\widetilde{b}_{F, e}^{Z Z}\right|$ & $<8 \times 10^{-9} \mathrm{GeV}^{-1}$ & Washington & {$[50]$} \\
$\left|\widetilde{b}_{F, e}^{(X Y)}\right|$ & $<2 \times 10^{-10} \mathrm{GeV}^{-1}$ & Harvard & {$[24]$} \\
$\left|\widetilde{b}_{F, e}^{(X Z)}\right|$ & $<1 \times 10^{-10} \mathrm{GeV}^{-1}$ & Harvard & {$[24]$} \\
$\left|\widetilde{b}_{F, e}^{(Y Z)}\right|$ & $<1 \times 10^{-10} \mathrm{GeV}^{-1}$ & Harvard & {$[24]$} \\
$\left|\widetilde{b}_{F, e}^{* X X}+\widetilde{b}_{F, e}^{* Y Y}\right|$ & $<2 \times 10^{-8} \mathrm{GeV}^{-1}$ & Washington & {$[50]$} \\
$\left|\widetilde{b}_{F, e}^{* X X}-\widetilde{b}_{F, e}^{* Y Y}\right|$ & $<4 \times 10^{-10} \mathrm{GeV}^{-1}$ & Harvard & {$[24]$} \\
$\left|\widetilde{b}_{F, e}^{* Z Z}\right|$ & $<8 \times 10^{-9} \mathrm{GeV}^{-1}$ & Washington & {$[50]$} \\
\hline
\end{tabular}

The measurement of the magnetic moment for positrons are currently under development at both Harvard University and Northwestern University [44,45]. A comparison with the results for that of electrons would offer opportunities to extract the coefficients that control only CPT-odd effects from the combination of coefficients in the difference (24). A sidereal-variation analysis of the positron anomaly frequencies would offer limits on the starred tilde coefficients $\widetilde{b}_{e}^{* J}, \widetilde{b}_{F, e}^{*(J K)}$ as well.

At the end of this subsection, we note that from Tables 4 and 5, for the 18 independent components of the tilde coefficients for Lorentz violation that are relevant to the $g$ factor measurements in Penning-trap experiments, only six of them in the proton sector and 12 of them in the electron sector have been constrained so far. Performing a full sidereal-variation analysis for the measurements data would permit access to the other components of the tilde coefficients.

\section{Summary}

In this work, we provide an overview of recent progress on searching for Lorentz- and CPT-violating signals using measurements of charge-to-mass ratios and magnetic moments in Penning-trap experiments. We first revisit the theory of Lorentz-violating quantum electrodynamics with operators of mass dimensions $d \leq 6$. The explicit expressions of the Lagrange density (1) are given in [21]. Perturbation theory is then applied to obtain the dominant energy shifts (6) and (10) for a confine particle and antiparticle due to Lorentz and CPT violation. This leads to the corresponding contributions to the cyclotron and anomaly frequencies in Equations (20) and (22). The results are then used to relate the coefficients for Lorentz violation to the experimental interpreted charge-to-mass ratio comparisons (23) between a particle and an antiparticle, as well as the magnetic moment comparisons (24). The general transformation of the related coefficients for Lorentz violation into different frames is performed using Equation (27). The explicit expressions relating the coefficients in the apparatus frame to the Sun-centered frame are given in Appendix A. The results show that for the charge-to-mass ratio comparisons between particles and antiparticles in Penning-trap experiments, the related coefficients for Lorentz violation in the Sun- 
centered frame are $\widetilde{b}_{w}^{\prime J}, \widetilde{c}_{w}^{(J K)}, \widetilde{b}_{w}^{J(K L)}, \widetilde{b}_{w}^{\prime * J}, \widetilde{c}_{w}^{*(J K)}$, and $\widetilde{b}_{w}^{* J(K L)}$. For experiments involving magnetic moment comparisons, the corresponding coefficients are $\widetilde{b}_{w}^{J}, \widetilde{b}_{w}^{* J}, \widetilde{b}_{F, w}^{(J K)}$, and $\widetilde{b}_{F, w}^{*(J K)}$. Using published results from existing Penning-trap experiments, constraints on various components of the coefficients for Lorentz violation are obtained. They are summarized in Tables 1-5. In conclusion, the high-precision measurements and excellent coverage of the coefficients for Lorentz violation offered by Penning-trap experiments provide strong motivations to continue the searches for possible Lorentz- and CPT-violating signals.

Author Contributions: Conceptualization, Y.D., T.D.O. and M.F.R.; methodology, Y.D., T.D.O. and M.F.R.; software, Y.D., T.D.O. and M.F.R.; validation, Y.D., T.D.O. and M.F.R.; formal analysis, Y.D., T.D.O. and M.F.R.; investigation, Y.D., T.D.O. and M.F.R.; resources, Y.D., T.D.O. and M.F.R.; data curation,Y.D., T.D.O. and M.F.R.; writing—original draft preparation,Y.D., T.D.O. and M.F.R.; writingreview and editing,Y.D., T.D.O. and M.F.R.; visualization, Y.D., T.D.O. and M.F.R.; supervision, Y.D., T.D.O. and M.F.R.; project administration, Y.D., T.D.O. and M.F.R.; funding acquisition, Y.D., T.D.O. and M.F.R..All authors have read and agreed to the published version of the manuscript.

Funding: This work was supported in part by the Gettysburg College Research and Professional Development Grant, the Gettysburg College Cross-Disciplinary Science Institute (X-SIG), and the Keck Grant from the W.M. Keck Science Department at Claremont McKenna, Pitzer, and Scripps Colleges.

Acknowledgments: The authors would like to thank Matthew Mewes for the invitation and Lisa Portmess for reading the manuscript.

Conflicts of Interest: The authors declare no conflict of interest.

\section{Appendix A. Transformations}

In this Appendix, we list the explicit expressions of the transformation results for the tilde coefficients for Lorentz violation that are relevant to the cyclotron and anomaly frequency shifts. These coefficients are $\widetilde{b}_{w}^{\prime 3}, \widetilde{c}_{w}^{11}+\widetilde{c}_{w}^{22}, \widetilde{b}_{w}^{311}+\widetilde{b}_{w}^{322}, \widetilde{b}_{w}^{3}, \widetilde{b}_{F, w}^{3}, \widetilde{b}_{w}^{\prime * 3}, \widetilde{c}_{w}^{* 11}+\widetilde{c}_{w}^{* 22}$, $\widetilde{b}_{w}^{* 311}+\widetilde{b}_{w}^{* 322}, \widetilde{b}_{w}^{* 3}$, and $\widetilde{b}_{F, w}^{* 3}$ in the apparatus frame.

We first consider Penning-trap experiments that use a vertical upward magnetic field. Taking $R^{a j}$ as the identity matrix and applying transformation (27) yield

$$
\widetilde{b}_{w}^{\prime 3}=\cos \omega_{\oplus} T_{\oplus} \widetilde{b}_{w}^{\prime X} \sin \chi+\sin \omega_{\oplus} T_{\oplus} \widetilde{b}_{w}^{\prime Y} \sin \chi+\widetilde{b}_{w}^{\prime Z} \cos \chi,
$$

$$
\begin{aligned}
\widetilde{c}_{w}^{11}+\widetilde{c}_{w}^{22}= & \cos 2 \omega_{\oplus} T_{\oplus}\left(-\frac{1}{2}\left(\widetilde{c}_{w}^{X X}-\widetilde{c}_{w}^{Y Y}\right) \sin ^{2} \chi\right)+\sin 2 \omega_{\oplus} T_{\oplus}\left(-\widetilde{c}_{w}^{(X Y)} \sin ^{2} \chi\right) \\
& +\cos \omega_{\oplus} T_{\oplus}\left(-\widetilde{c}_{w}^{(X Z)} \sin 2 \chi\right)+\sin \omega_{\oplus} T_{\oplus}\left(-\widetilde{c}_{w}^{(Y Z)} \sin 2 \chi\right) \\
& +\frac{1}{4}\left(\widetilde{c}_{w}^{X X}+\widetilde{c}_{w}^{Y Y}\right)(3+\cos 2 \chi)+\widetilde{c}_{w}^{Z Z} \sin ^{2} \chi
\end{aligned}
$$

$$
\begin{aligned}
\widetilde{b}_{w}^{311}+\widetilde{b}_{w}^{322}= & \cos 3 \omega_{\oplus} T_{\oplus}\left(\left[-\frac{1}{4}\left(\widetilde{b}_{w}^{X X X}-\widetilde{b}_{w}^{X Y Y}\right)+\frac{1}{2} \widetilde{b}_{w}^{Y(X Y)}\right] \sin ^{3} \chi\right) \\
+ & \sin 3 \omega_{\oplus} T_{\oplus}\left(\left[-\frac{1}{2} \widetilde{b}_{w}^{X(X Y)}-\frac{1}{4}\left(\widetilde{b}_{w}^{Y X X}-\widetilde{b}_{w}^{Y Y Y}\right)\right] \sin ^{3} \chi\right) \\
+ & \cos 2 \omega_{\oplus} T_{\oplus}\left(\left[-\widetilde{b}_{w}^{X(X Z)}+\widetilde{b}_{w}^{Y(Y Z)}-\frac{1}{2}\left(\widetilde{b}_{w}^{Z X X}-\widetilde{b}_{w}^{Z Y Y}\right)\right] \cos \chi \sin ^{2} \chi\right) \\
+ & \sin 2 \omega_{\oplus} T_{\oplus}\left(\left[-\widetilde{b}_{w}^{X(Y Z)}-\widetilde{b}_{w}^{Y(X Z)}-\widetilde{b}_{w}^{Z(X Y)}\right] \cos \chi \sin ^{2} \chi\right) \\
+ & \cos \omega_{\oplus} T_{\oplus}\left(\frac{1}{8} \widetilde{b}_{w}^{X X X}(5+3 \cos 2 \chi) \sin \chi+\frac{1}{8} \widetilde{b}_{w}^{X Y Y}(7+\cos 2 \chi) \sin \chi+\widetilde{b}_{w}^{X Z Z} \sin ^{3} \chi\right. \\
& \left.-\frac{1}{2} \widetilde{b}_{w}^{Y(X Y)} \sin ^{3} \chi-2 \widetilde{b}_{w}^{Z(X Z)} \cos ^{2} \chi \sin \chi\right) \\
+ & \sin \omega_{\oplus} T_{\oplus}\left(-\frac{1}{2} \widetilde{b}_{w}^{X(X Y)} \sin ^{3} \chi+\frac{1}{8} \widetilde{b}_{w}^{Y X X}(7+\cos 2 \chi) \sin \chi+\frac{1}{8} \widetilde{b}_{w}^{Y Y Y}(5+3 \cos 2 \chi) \sin \chi\right. \\
& \left.+\widetilde{b}_{w}^{Y Z Z} \sin ^{3} \chi-2 \widetilde{b}_{w}^{Z(Y Z)} \cos ^{2} \chi \sin \chi\right)
\end{aligned}
$$




$$
\begin{array}{r}
-\left(\widetilde{b}_{w}^{X(X Z)}+\widetilde{b}_{w}^{Y(Y Z)}-\widetilde{b}_{w}^{Z Z Z}\right) \cos \chi \sin ^{2} \chi+\left(\widetilde{b}_{w}^{Z X X}+\widetilde{b}_{w}^{Z Y Y}\right) \cos \chi \cos 2 \chi, \\
\widetilde{b}_{w}^{3}=\cos \omega_{\oplus} T_{\oplus} \widetilde{b}_{w}^{X} \sin \chi+\sin \omega_{\oplus} T_{\oplus} \widetilde{b}_{w}^{Y} \sin \chi+\widetilde{b}_{w}^{Z} \cos \chi,
\end{array}
$$

and

$$
\begin{aligned}
\widetilde{b}_{F, w}^{33}= & \left.\cos 2 \omega_{\oplus} T_{\oplus}\left(\frac{1}{2}\left(\widetilde{b}_{F, w}^{X X}-\widetilde{b}_{F, w}^{Y Y}\right) \sin ^{2} \chi\right)+\sin 2 \omega_{\oplus} T_{\oplus} \widetilde{b}_{F, w}^{(X Y)} \sin ^{2} \chi\right) \\
& +\cos \omega_{\oplus} T_{\oplus} \widetilde{b}_{F, w}^{(X Z)} \sin 2 \chi+\sin \omega_{\oplus} T_{\oplus} \widetilde{b}_{F, w}^{(Y Z)} \sin 2 \chi \\
& +\frac{1}{2}\left(\widetilde{b}_{F, w}^{X X}+\widetilde{b}_{F, w}^{Y Y}-2 \widetilde{b}_{F, w}^{Z Z}\right) \sin ^{2} \chi+\widetilde{b}_{F, w}^{Z Z} .
\end{aligned}
$$

We then switch to the case where a horizontal magnetic field is used in the trap, directing at an angle $\theta$ from the local south in the counterclockwise direction. This implies that the Euler angles $(\alpha, \beta, \gamma)=(\theta, \pi / 2,0)$. Applying the transformation (27) we have

$$
\begin{aligned}
\widetilde{b}_{w}^{\prime 3}= & \cos \omega_{\oplus} T_{\oplus}\left(\widetilde{b}_{w}^{\prime X} \cos \theta \cos \chi+\widetilde{b}_{w}^{\prime Y} \sin \theta\right)+\sin \omega_{\oplus} T_{\oplus}\left(-\widetilde{b}_{w}^{\prime X} \sin \theta+\widetilde{b}_{w}^{\prime Y} \cos \theta \cos \chi\right) \\
& -\widetilde{b}_{w}^{\prime Z} \cos \theta \sin \chi,
\end{aligned}
$$

$$
\begin{aligned}
\widetilde{c}_{w}^{11}+\widetilde{c}_{w}^{22}= & \cos 2 \omega_{\oplus} T_{\oplus}\left(\frac{1}{8}\left(\widetilde{c}_{w}^{X X}-\widetilde{c}_{w}^{Y Y}\right)\left(1-3 \cos 2 \theta-2 \cos ^{2} \theta \cos 2 \chi\right)-\widetilde{c}_{w}^{(X Y)} \cos \chi \sin 2 \theta\right) \\
& +\sin 2 \omega_{\oplus} T_{\oplus}\left(\frac{1}{2}\left(\widetilde{c}_{w}^{X X}-\widetilde{c}_{w}^{Y Y}\right) \cos \chi \sin 2 \theta+\frac{1}{4} \widetilde{c}_{w}^{(X Y)}\left(1-3 \cos 2 \theta-2 \cos ^{2} \theta \cos 2 \chi\right)\right) \\
& +\cos \omega_{\oplus} T_{\oplus}\left(\widetilde{c}_{w}^{(X Z)} \cos ^{2} \theta \sin 2 \chi+\widetilde{c}_{w}^{(Y Z)} \sin 2 \theta \sin \chi\right) \\
& +\sin \omega_{\oplus} T_{\oplus}\left(-\widetilde{c}_{w}^{(X Z)} \sin 2 \theta \sin \chi+\widetilde{c}_{w}^{(Y Z)} \cos ^{2} \theta \sin 2 \chi\right) \\
& +\frac{1}{2}\left(\widetilde{c}_{w}^{X X}+\widetilde{c}_{w}^{Y Y}\right)\left(\cos ^{2} \theta+\cos ^{2} \chi \sin ^{2} \theta+\sin ^{2} \chi\right)+\widetilde{c}_{w}^{Z Z}\left(\cos ^{2} \chi+\sin ^{2} \theta \sin ^{2} \chi\right),
\end{aligned}
$$

$$
\begin{aligned}
& \widetilde{b}_{w}^{311}+\widetilde{b}_{w}^{322} \\
& =\cos 3 \omega_{\oplus} T_{\oplus}\left(\left[\frac{1}{64}\left(\widetilde{b}_{w}^{X X X}-\widetilde{b}_{w}^{X Y Y}\right)-\frac{1}{32} \widetilde{b}_{w}^{Y(X Y)}\right]\left[3(\cos \theta-5 \cos 3 \theta) \cos \chi-4 \cos ^{3} \theta \cos 3 \chi\right]\right. \\
& \left.+\left[\frac{1}{16} \widetilde{b}_{w}^{X(X Y)}+\frac{1}{32}\left(\widetilde{b}_{w}^{Y X X}-\widetilde{b}_{w}^{Y Y Y}\right)\right]\left[3 \sin \theta\left(1-4 \cos ^{2} \theta \cos 2 \chi\right)-5 \sin 3 \theta\right]\right) \\
& +\sin 3 \omega_{\oplus} T_{\oplus}\left(\left[-\frac{1}{32}\left(\widetilde{b}_{w}^{X X X}-\widetilde{b}_{w}^{X Y Y}\right)+\frac{1}{16} \widetilde{b}_{w}^{Y(X Y)}\right]\left[3 \sin \theta\left(1-4 \cos ^{2} \theta \cos 2 \chi\right)-5 \sin 3 \theta\right]\right. \\
& \left.+\left[\frac{1}{32} \widetilde{b}_{w}^{X(X Y)}+\frac{1}{64}\left(\widetilde{b}_{w}^{Y X X}-\widetilde{b}_{w}^{Y Y Y}\right)\right]\left[3(\cos \theta-5 \cos 3 \theta) \cos \chi-4 \cos ^{3} \theta \cos 3 \chi\right]\right) \\
& +\cos 2 \omega_{\oplus} T_{\oplus}\left([ \frac { 1 } { 1 6 } \widetilde { b } _ { w } ^ { X ( X Z ) } - \frac { 1 } { 1 6 } \widetilde { b } _ { w } ^ { Y ( Y Z ) } + \frac { 1 } { 3 2 } ( \widetilde { b } _ { w } ^ { Z X X } - \widetilde { b } _ { w } ^ { Z Y Y } ) ] \left[\left(5 \cos 3 \theta-\cos \theta \sin \chi+4 \cos ^{3} \theta \sin 3 \chi\right]\right.\right. \\
& \left.+\left(\widetilde{b}_{w}^{X(Y Z)}+\widetilde{b}_{w}^{Y(X Z)}+\widetilde{b}_{w}^{Z(X Y)}\right) \sin \theta \cos ^{2} \theta \sin 2 \chi\right) \\
& +\sin 2 \omega_{\oplus} T_{\oplus}\left(\left[-\widetilde{b}_{w}^{X(X Z)}+\widetilde{b}_{w}^{Y(Y Z)}-\frac{1}{2}\left(\widetilde{b}_{w}^{Z X X}-\widetilde{b}_{w}^{Z Y Y}\right)\right] \sin \theta \cos ^{2} \theta \sin 2 \chi\right. \\
& \left.-\left(\frac{1}{16} \widetilde{b}_{w}^{X(Y Z)}+\frac{1}{16} \widetilde{b}_{w}^{Y(X Z)}+\frac{1}{16} \widetilde{b}_{w}^{Z(X Y)}\right)\left[(\cos \theta-5 \cos 3 \theta) \sin \chi-4 \cos ^{3} \theta \sin 3 \chi\right]\right) \\
& +\cos \omega_{\oplus} T_{\oplus}\left(\frac{1}{16} \widetilde{b}_{w}^{X X X} \cos \theta \cos \chi\left(-6 \cos ^{2} \theta \cos 2 \chi+3 \cos 2 \theta+7\right)\right. \\
& -\frac{1}{2} \widetilde{b}_{w}^{X(X Y)} \sin \theta\left(\cos ^{2} \theta \cos 2 \chi+\sin ^{2} \theta \cos ^{2} \chi+\sin ^{2} \chi\right) \\
& +\frac{1}{16} \widetilde{b}_{w}^{X Y Y} \cos \theta \cos \chi\left(-2 \cos ^{2} \theta \cos 2 \chi+\cos 2 \theta+13\right) \\
& +\widetilde{b}_{w}^{X Z Z} \cos \theta \cos \chi\left(\sin ^{2} \theta \sin ^{2} \chi+\cos ^{2} \chi\right) \\
& +\frac{1}{32} \widetilde{b}_{w}^{Y X X}\left[\sin \theta\left(25-4 \cos ^{2} \theta \cos 2 \chi\right)+\sin 3 \theta\right]
\end{aligned}
$$




$$
\begin{aligned}
& +\frac{1}{16} \widetilde{b}_{w}^{Y(X Y)} \cos \theta\left[(\cos 2 \theta-7) \cos \chi-2 \cos ^{2} \theta \cos 3 \chi\right] \\
& +\frac{1}{32} \widetilde{b}_{w}^{Y Y Y}\left[\sin \theta\left(11-12 \cos ^{2} \theta \cos 2 \chi\right)+3 \sin 3 \theta\right] \\
& +\widetilde{b}_{w}^{Y Z Z} \sin \theta\left(\sin ^{2} \theta \sin ^{2} \chi+\cos ^{2} \chi\right) \\
& \left.-2 \widetilde{b}_{w}^{Z(X Z)} \cos ^{3} \theta \sin ^{2} \chi \cos \chi-2 \widetilde{b}_{w}^{Z(Y Z)} \sin \theta \cos ^{2} \theta \sin ^{2} \chi\right) \\
& +\sin \omega_{\oplus} T_{\oplus}\left(\frac{1}{32} \widetilde{b}_{w}^{X X X}\left[\sin \theta\left(12 \cos ^{2} \theta \cos 2 \chi-11\right)-3 \sin 3 \theta\right]\right. \\
& +\frac{1}{16} \widetilde{b}_{w}^{X(X Y)} \cos \theta\left[(\cos 2 \theta-7) \cos \chi-2 \cos ^{2} \theta \cos 3 \chi\right] \\
& +\frac{1}{32} \widetilde{b}_{w}^{X Y Y}\left[\sin \theta\left(4 \cos ^{2} \theta \cos 2 \chi-25\right)-\sin 3 \theta\right] \\
& -\widetilde{b}_{w}^{X Z Z} \sin \theta\left(\sin ^{2} \theta \sin ^{2} \chi+\cos ^{2} \chi\right) \\
& +\frac{1}{16} \widetilde{b}_{w}^{Y X X} \cos \theta \cos \chi\left(-2 \cos ^{2} \theta \cos 2 \chi+\cos 2 \theta+13\right) \\
& +\frac{1}{2} \widetilde{b}_{w}^{Y(X Y)} \sin \theta\left(\cos ^{2} \theta \cos 2 \chi+\sin ^{2} \theta \cos ^{2} \chi+\sin ^{2} \chi\right) \\
& +\frac{1}{16} \widetilde{b}_{w}^{Y Y Y} \cos \theta \cos \chi\left(-6 \cos ^{2} \theta \cos 2 \chi+3 \cos 2 \theta+7\right) \\
& +\widetilde{b}_{w}^{Y Z Z} \cos \theta \cos \chi\left(\sin ^{2} \theta \sin ^{2} \chi+\cos ^{2} \chi\right) \\
& \left.+2 \widetilde{b}_{w}^{Z(X Z)} \sin \theta \cos ^{2} \theta \sin ^{2} \chi-2 \widetilde{b}_{w}^{Z(Y Z)} \cos ^{3} \theta \sin ^{2} \chi \cos \chi\right) \\
& +\frac{1}{8}\left(\widetilde{b}_{w}^{X(X Z)}+\widetilde{b}_{w}^{Y(Y Z)}-\widetilde{b}_{w}^{Z Z Z}\right) \cos \theta\left(-4 \cos 2 \theta \sin ^{3} \chi+5 \sin \chi+\sin 3 \chi\right) \\
& +\frac{1}{16}\left(\widetilde{b}_{w}^{Z X X}+\widetilde{b}_{w}^{Z Y Y}\right)\left[2 \cos ^{3} \theta \sin 3 \chi-\cos \theta \sin \chi(3 \cos 2 \theta+11)\right] \text {, } \\
& \widetilde{b}_{w}^{3}=\cos \omega_{\oplus} T_{\oplus}\left(\widetilde{b}_{w}^{X} \cos \theta \cos \chi+\widetilde{b}_{w}^{Y} \sin \theta\right)+\sin \omega_{\oplus} T_{\oplus}\left(-\widetilde{b}_{w}^{X} \sin \theta+\widetilde{b}_{w}^{Y} \cos \theta \cos \chi\right) \\
& -\widetilde{b}_{w}^{Z} \cos \theta \sin \chi \text {, } \\
& \text { and } \\
& \widetilde{b}_{F, w}^{33}=\cos 2 \omega_{\oplus} T_{\oplus}\left(\frac{1}{2}\left(\widetilde{b}_{F, w}^{X X}-\widetilde{b}_{F, w}^{Y Y}\right)\left(\cos ^{2} \theta \cos ^{2} \chi-\sin ^{2} \theta\right)+\widetilde{b}_{F, w}^{(X Y)} \sin 2 \theta \cos \chi\right) \\
& +\sin 2 \omega_{\oplus} T_{\oplus}\left(\frac{1}{4} \widetilde{b}_{F, w}^{(X Y)}\left(2 \cos ^{2} \theta \cos 2 \chi+3 \cos 2 \theta-1\right)-\left(\widetilde{b}_{F, w}^{X X}-\widetilde{b}_{F, w}^{Y Y}\right) \sin 2 \theta \cos \chi\right) \\
& +\cos \omega_{\oplus} T_{\oplus}\left(-2 \widetilde{b}_{F, w}^{(X Z)} \cos ^{2} \theta \cos \chi-\widetilde{b}_{F, w}^{(Y Z)} \sin 2 \theta \sin \chi\right) \\
& +\sin \omega_{\oplus} T_{\oplus}\left(\widetilde{b}_{F, w}^{(X Z)} \sin 2 \theta \sin \chi-2 \widetilde{b}_{F, w}^{(Y Z)} \cos ^{2} \theta \cos \chi\right) \\
& +\frac{1}{2}\left(\widetilde{b}_{F, w}^{X X}+\widetilde{b}_{F, w}^{Y Y}\right)\left(\cos ^{2} \theta \cos ^{2} \chi+\sin ^{2} \theta\right)+\widetilde{b}_{F, w}^{Z Z} \cos ^{2} \theta \sin ^{2} \chi .
\end{aligned}
$$

The corresponding transformations for the starred tilde quantities $\widetilde{b}_{w}^{\prime * 3}, \widetilde{c}_{w}^{* 11}+\widetilde{c}_{w}^{* 22}$, $\widetilde{b}_{w}^{* 311}+\widetilde{b}_{w}^{* 322}, \widetilde{b}_{w}^{* 3}$, and $\widetilde{b}_{F, w}^{33}$ take the same forms as these given above, with the substitutions of the usual tilde coefficients by the starred ones.

\section{References}

1. Hanneke, D.; Fogwell, S.; Gabrielse, G. New measurement of the electron magnetic moment and the fine structure constant. Phys. Rev. Lett. 2008, 100, 120801. [CrossRef] [PubMed]

2. Kostelecký, V.A.; Samuel, S. Spontaneous breaking of Lorentz symmetry in string theory. Phys. Rev. D 1989, 39, 683. [CrossRef] [PubMed]

3. Kostelecký, V.A.; Potting, R. CPT and strings. Nucl. Phys. B 1991 359, 545-570. [CrossRef]

4. Colladay, D.; Kostelecký, V.A. CPT violation and the Standard Model. Phys. Rev. D 1997, 55, 6760. [CrossRef]

5. Colladay, D.; Kostelecký, V.A. Lorentz-violating extension of the Standard Model. Phys. Rev. D 1998, 58, 116002. [CrossRef]

6. Greenberg, O.W. CPT violation implies violation of Lorentz invariance. Phys. Rev. Lett. 2002, 89, 231602. [CrossRef] [PubMed]

7. Kostelecký, V.A.; Russell, N. Data tables for Lorentz and CPT violation. Rev. Mod. Phys. 2011, 83, 11. [CrossRef]

8. Kostelecký, V.A. Gravity, Lorentz violation, and the Standard Model. Phys. Rev. D 2004, 69, 105009. [CrossRef]

9. Kostelecký, V.A.; Lehnert, R. Stability, causality, and Lorentz and CPT violation. Phys. Rev. D 2001, 63, 065008. [CrossRef]

10. Drummond, I.T. Quantum field theory in a multimetric background. Phys. Rev. D 2013, 88, 025009. [CrossRef] 
11. Belich, H; Bernald, L.D.; Gaete, P; Helayël-Neto, J.A.; Leal, F.J.L. Aspects of CPT-even Lorentz-symmetry violating physics in a supersymmetric scenario. Eur. Phys. J. C 2015, 75, 291. [CrossRef]

12. Carroll, S.M.; Harvey, J.A.; Kostelecký, V.A.; Lane, C.D.; Okamoto, T. Noncommutative field theory and Lorentz violation. Phys. Rev. Lett. 2001, 87, 141601. [CrossRef]

13. Chaichian, M.; Sheikh-Jabbari, M.M.; Tureanu, A. Non-commutativity of space-time and the hydrogen atom spectrum. Eur. Phys. J. C 2004, 36, 251-252. [CrossRef]

14. Hayakawa, M. Perturbative analysis on infrared aspects of noncommutative QED on $R^{4}$. Phys. Lett. B 2000, 478, 394-400. [CrossRef]

15. Kostelecký, V.A. Riemann-Finsler geometry and Lorentz-violating kinematics. Phys. Lett. B 2011, 701, 137-143. [CrossRef]

16. Silva, J.E.G.; Maluf, R.V.; Almeida, C.A.S. A nonlinear dynamics for the scalar field in Randers spacetime. Phys. Lett. B 2017, 766, 263-267. [CrossRef]

17. Foster, J.; Lehnert, R. Classical-physics applications for Finsler $b$ space. Phys. Lett. B 2015, 746, 164-170. [CrossRef]

18. Edwards, B.; Kostelecký, V.A. Riemann-Finsler geometry and Lorentz-violating scalar fields. Phys. Lett. B 2018, 786, 319-326. [CrossRef]

19. Bluhm, R.; Kostelecký, V.A.; Russell, N. Testing CPT with anomalous magnetic moments. Phys. Rev. Lett. 1997, 79, 1432. [CrossRef]

20. Bluhm, R.; Kostelecký, V.A.; Russell, N. CPT and Lorentz tests in Penning traps. Phys. Rev. D 1998, 57, 3932. [CrossRef]

21. Ding, Y.; Kostelecký, V.A. Lorentz-violating spinor electrodynamics and Penning traps. Phys. Rev. D 2016, 94, 056008. [CrossRef]

22. Ding, Y.; Rawnak, M.F. Lorentz and CPT tests with charge-to-mass ratio comparisons in Penning traps. Phys. Rev. D 2020, 94, 056009. [CrossRef]

23. Mittleman, R.K.; Ioannou, I.I.; Dehmelt, H.G.; Russell, N. Bound on CPT and Lorentz symmetry with a trapped electron. Phys. Rev. Lett. 1999, 102, 2116. [CrossRef]

24. Hanneke, D. Cavity Control in a Single-Electron Quantum Cyclotron: An Improved Measurement of the Electron Magnetic Moment. Ph.D. Thesis, Harvard University, Cambridge, MA, USA, 2007.

25. Ding, Y. Lorentz and CPT tests using Penning traps. Symmetry 2019, 11, 1220. [CrossRef]

26. Bluhm, R.; Kostelecký, V.A.; Lane, C.D. CPT and Lorentz tests with muons. Phys. Rev. Lett. 2000, 84, 1098. [CrossRef] [PubMed]

27. Gomes, A.H.; Kostelecký, V.A.; Vargas, A. Laboratory tests of Lorentz and CPT symmetry with muons. Phys. Rev. D 2014, 90, 076009. [CrossRef]

28. Kostelecký, V.A.; Vargas, A. Lorentz and CPT tests in hydrogen, antihydrogen, and related systems. Phys. Rev. D 2015, 92, 056002. [CrossRef]

29. Kostelecký, V.A.; Vargas, A. Lorentz and CPT Tests with clock-comparison experiments. Phys. Rev. D 2018, 98, 036003. [CrossRef]

30. Kostelecký, V.A.; Mewes, M. Fermions with Lorentz-violating operators of arbitrary dimension. Phys. Rev. D 2013, 88, 096006. [CrossRef]

31. Li, Z.; Kostelecký, V.A. Gauge field theories with Lorentz-violating operators of arbitrary dimension. Phys. Rev. D 2019, 99, 056016.

32. Kostelecký, V.A.; Mewes, M. Electrodynamics with Lorentz-violating operators of arbitrary dimension. Phys. Rev. D 2009, 80, 015020. [CrossRef]

33. Kostelecký, V.A.; Mewes, M. Neutrinos with Lorentz-violating operators of arbitrary dimension. Phys. Rev. D 2012, 85, 096005. [CrossRef]

34. Kostelecký, V.A.; Mewes, M.; Testing local Lorentz invariance with gravitational waves. Phys. Lett. B 2016, 757, 510-514. [CrossRef]

35. For a review see, for example, Brown, L.S.; Gabrielse, G. Geonium theory: Physics of a single electron or ion in a Penning trap. Rev. Mod. Phys. 1986, 58, 233.

36. Kostelecký, V.A.; Lane, C.D. Constraints on Lorentz violation from clock-comparison experiments. Phys. Rev. D 1999, 60, 116010. [CrossRef]

37. Kostelecký, V.A.; Mewes, M. Signals for Lorentz violation in electrodynamics. Phys. Rev. D 2002, 66, 056005. [CrossRef]

38. Canè, F.; Bear, D.; Phillips, D.F.; Rosen, M.S.; Smallwood, C.L.; Stoner, R.E.; Walsworth, R.L.; Kostelecký, V.A. Bound on Lorentz and CPT violating boost effects for the neutron. Phys. Rev. Lett. 2004, 93, 230801. [CrossRef] [PubMed]

39. Heckel, B.R.; Adelberger, E.G.; Cramer, C.E.; Cook, T.S.; Schlamminger, S.; Schmidt, U. Preferred-frame and CP-violation tests with polarized electrons. Phys. Rev. D 2008, 78, 092006. [CrossRef]

40. Bluhm, R.; Kostelecký, V.A.; Lane, C.D.; Russell, N. Probing Lorentz and CPT violation with space-based experiments. Phys. Rev. D 2003, 68, 125008. [CrossRef]

41. Gabrielse, G.; Khabbaz, A.; Hall, D.S.; Heimann, C.; Kalinowsky, H.; Jhe, W. Precision mass spectroscopy of the antiproton and proton using simultaneously trapped particles. Phys. Rev. Lett. 1999, 82, 3198. [CrossRef]

42. Ulmer, S.; Smorra, C.; Mooser, A.; Franke, K.; Nagahama, H.; Schneider, G.; Higuchi, T.; Van Gorp S.; Blaum, K.; Matsuda, Y.; et al. High-precision comparison of the antiproton-to-proton charge-to-mass ratio. Nature 2015, 524, 196-199. [CrossRef]

43. Schwinberg, P.B.; Van Dyck Jr. R.S.; Dehmelt H.G. Trapping and thermalization of positrons for geonium spectroscopy. Phys. Lett. A 1981, 81, 119-120. [CrossRef]

44. Hoogerheide, S.F.; Dorr, J.C.; Novitski, E.; Gabrielse, G. High efficiency positron accumulation for high-precision magnetic moment experiments. Rev. Sci. Instrum. 2015, 86, 053301. [CrossRef]

45. Gabrielse, G.; Fayer, S.E.; Myers, T.G.; Fan, X. Towards an improved test of the Standard Model's most precise prediction. Atoms 2019, 7, 45. [CrossRef] 
46. Schneider, G.; Mooser, A.; Bohman, M.; Schön, N.; Harrington, J.; Higuchi, T.; Nagahama, H.; Sellner, S.; Smorra, C.; Blaum, K.; et al. Double-trap measurement of the proton magnetic moment at 0.3 parts per billion precision. Science 2017, 358, 1081-1084. [CrossRef]

47. Mooser, A.; Ulmer, S.; Blaum, K.; Franke, K.; Kracke, H.; Leiteritz, C.; Quint, W.; Rodegheri, C.C.; Smorra, C.; Walz, J. Direct high-precision measurement of the magnetic moment of the proton. Nature 2014, 509, 596-599. [CrossRef]

48. Smorra, C.; Sellner, S.; Borchert, M.J.; Harrington, J.A.; Higuchi, T.; Nagahama, H.; Tanaka, T.; Mooser, A.; Schneider, G.; Bohman, M.; et al. A parts-per-billion measurement of the antiproton magnetic moment. Nature 2017, 550, 371-374. [CrossRef] [PubMed]

49. Meiners, T.; Niemann, M.; Paschke, A.G.; Borchert, M.; Idel, A.; Mielke, J.; Voges, K.; Bautista-Salvador, A.; Lehnert, R.; Ulmer, S.; et al. Towards sympathetic laser cooling and detection of single (anti-)protons. In Proceedings of the Seventh Meeting on CPT and Lorentz Symmetry; Kostelecký, V.A., Ed.; World Scientific: Singapore, 2017.

50. Dehmelt, H.G.; Mittleman, R.K.; Van Dyck R.S.; Schwinberg, P. Past electron-positron $g-2$ experiments yielded sharpest bound on CPT violation for point particles. Phys. Rev. Lett. 1999, 83, 4694. [CrossRef] 